$$
\text { doi:10.13108/2021-13-3-126 }
$$

\title{
ON SINGULARITY STRUCTURE OF MINIMAX SOLUTION TO DIRICHLET PROBLEM FOR EIKONAL TYPE EQUATION WITH DISCONTINUOUS CURVATURE OF BOUNDARY OF BOUNDARY SET
}

\author{
A.A. USPENSKII, P.D. LEBEDEV
}

\begin{abstract}
The birth of nonsmooth singularities in the minimax (generalized) solution of the Dirichlet problem for the eikonal equation is due to the existence of pseudo-vertices, the singular points of the boundary of the boundary set. Finding the pseudo-vertices is the first step in the procedure for constructing a singular set for solving a boundary value problem. To find these points, one has to construct local solutions to an equation of the golden ratio type, which establishes a connection between the eikonal operator and the geometry of the boundary set. The problem of identifying local solutions to the equation is related to the problem of finding fixed points of the mappings formed by local reparametrization of the boundary of the boundary set. In this work we obtain necessary conditions for the existence of pseudo-vertices when the smoothness of the curvature of a parametrically given boundary of the boundary set is broken. The conditions are written in various equivalent forms. In particular, we obtain a representation in the form of a convex combination of one-sided derivatives of the curvature. We provide the formulae for the coefficients of a convex combination determined by markers, which scalar characteristics of the pseudovertices. We find an algebraic equation, the roots of which are the markers. We adduce an example of the numerical-analytical construction of a minimax solution to the Dirichlet problem and this example demonstrates the effectiveness of the developed methods for solving nonsmooth boundary value problems.
\end{abstract}

Keywords: first order partial differential equation, minimax solution, speed, wavefront, diffeomorphism, eikonal, optimal result function, singular set, symmetry, pseudo-vertex.

Mathematics Subject Classification: 32S30, 32T27, 34H20, 35A18, 35B32

\section{INTRODUCTION}

First order partial differential equations, to which the eikonal equation belongs, model the processes in mechanics, geometric optics, optimal control theory, differential games, seismology, economics, and other branches of science and applications. The problem on existence of a classical solution in the class of continuously differentiable functions and the uniqueness problem are overcome by introducing generalized solutions, which are already considered, as a rule, on the set of continuous functions, and sometimes on the set of discontinuous functions, see [1]-[4].

The concept of a minimax solution [4] is based on constructions from the theory of positional differential games [5] and give an opportunity to develop theoretical approaches and effective

A.A. Uspenskit, P.D. Lebedev, On singularity structure of minimax solution to Dirichlet PROBLEM FOR EIKONAL TYPE EQUATION WITH DISCONTINUOUS CURVATURE OF BOUNDARY OF BOUNDARY SET.

(C) Uspenskil A.A., LeBedev P.D. 2021.

The work is made in the framework of researches carried out in Ural Mathematical Center under the financial support of the Ministry of Science and Higher Education of Russian Federation (agreement no. 075-02-20211383).

Submitted July 2, 2020. 
numerical procedures for constructing solutions to various classes of boundary value problems for first order partial differential equation and Hamiltonian equations considered, in particular, the control theory and theory of differential games, see, for instance, [6] [ 8].

In the present work we study a problem on emerging singularity of a minimax solution to Hamilton-Jacobi equation; this solution is a function of optimal result in a corresponding speed control problem. A non-convexity of the boundary set gives rise, even for a sufficiently high order of differentiability of its boundary, to the loss of smoothness of the minimax solution. We study the case, when the boundary of the boundary set is twice continuously differentiable but there are points, at which the curvature looses the smoothness. The construction of the generalized solution begins with finding out a singular set, on which the gradient of the solution has a discontinuity. The methods and constructive approaches for constructing singular sets, as well as analytic and numerical procedures on their base for constructing a solution to the Dirichlet problem for an eikonal type equation were proposed in [9]-[15].

A key relation of the developed theory is an equation of a golden ratio type, the solution of which determine pseudovertices. The main result of the present work is a theorem on necessary conditions of existence of pseudo-vertices, which are special points on the boundary of the boundary set, related with characterizing the boundary set from the point of view of measuring its non-convexity [16]. The conditions are obtained in a non-classical form as stationarity conditions, formulated in terms of one-sided limits, for a function of two variables determining the main part of the golden ration type. Moreover, the necessary conditions are found in form relating the constructions of the smooth analysis with those of the convex analysis, namely, in form of a convex combination of one-sided third order derivatives. The important elements of constructions are the relations for determining the coefficients of the convex combination, which are functions of one-sided markers of the pseudo-vertices. At that, the markers are calculated as the roots of a third order polynomial with the coefficients by found laws in terms of one-sided derivatives of the curvature.

The obtained theoretical results are illustrated by an example of constructing a minimax solution to a Dirichlet boundary value problem; this solution is a function of the optimal result for a corresponding speed control problem.

\section{OBJECT OF STUDY}

We consider the Dirichlet problem for the Hamilton-Jacobi equation:

$$
\min _{\nu:\|\nu\| \leqslant 1}\left(\nu_{1} \frac{\partial u}{\partial x_{1}}+\nu_{2} \frac{\partial u}{\partial x_{2}}\right)+1=0,\left.\quad u\right|_{\Gamma}=0 .
$$

Here $\|\nu\|=\sqrt{\nu_{1}^{2}+\nu_{2}^{2}}$ is the norm of the vector $\nu=\left(\nu_{1}, \nu_{2}\right)$. The boundary condition in (2.1) is imposed on the boundary $\Gamma=\partial M$ of a closed set $M \subset \mathbb{R}^{2}$. The curve $\Gamma$ has no self-intersection points. Differential properties of $\Gamma$ influence essentially the structure of the minimax solution of problem (2.1); we provide their complete list below while justifying the statements.

The minimax solution of problem 2.1] $u(\mathbf{x})=\rho(\mathbf{x}, M)$ [9], where $\rho(\mathbf{x}, M)=\inf _{\mathbf{m} \in M}\|\mathbf{x}-\mathbf{m}\|$ is the Euclidean distance from a point $\mathbf{x}=\left(x_{1}, x_{2}\right)$ to the set $M$, is the function of an optimal result in speed control problem with a simple dynamics:

$$
\left\{\begin{array}{l}
\dot{x}_{1}=\nu_{1} \\
\dot{x}_{2}=\nu_{2}
\end{array}\right.
$$

where the control $\nu=\left(\nu_{1}, \nu_{2}\right)$ is restricted by the constraint $\|\nu\| \leqslant 1, M$ is a target set. Moreover, a minimax solution of problem (2.1) taken with an opposite sign coincides with the fundamental (in the sense of S.N. Kruzhkov) solution $u=u_{k}(x, y)$ [2] of the Dirichlet problem 
for the eikonal equation in the case of an isotropic media:

$$
\left(\frac{\partial u}{\partial x_{1}}\right)^{2}+\left(\frac{\partial u}{\partial x_{2}}\right)^{2}=1,\left.\quad u\right|_{\Gamma}=0 .
$$

Here the boundary condition is the same as in problem (2.1). The level line maps of the fundamental solution of problem (2.3) and of the minimax solution of problem (2.1) coincide. In this sense the solutions of both boundary value problems are equivalent. The character of the evolution of the wavefronts and of their singularities is determined by the geometry of the boundary set and by the differential property of its boundary. The non-convexity of this set produces the presence of a singular set for the solution of this problem, which in the general case consists of zero- and one-dimensional manifolds. Both in analytic and numerical construction of the minimax solution to problem (2.1), a special role is played by pseudo-vertices, which are the points on the boundary of the boundary set producing the branches of the singular set.

We mention that it is easiest to find the pseudo-vertices for two classes of curves enveloping the boundary set. The first class is formed by piece-wise smooth curves, the corner points of which are pseudo-vertices [12. The second class is the curves with the smoothness of order at least three, the pseudo-vertices of which are contained in the set of the points with stationary curvature [13]. The most complicated for the analysis curves are ones with of a varying smoothness, the order of which can locally vary from 1 to 2 . For the case, when the smoothness of the curve is minimal, that is, is equal 1, we obtain necessary conditions for the existence of pseudo-vertices of the boundary set in terms of one-sided partial limits of the differential relations depending on the properties of diffeomorphisms determining the pseudo-vertices [14]. In the present work we study one of the intermediate cases, in which at the points of a parametrically defined curve $\Gamma$, a classical curvature is well-defined, but at the same time, there can be a discontinuity of the third derivatives of the coordinate functions.

\section{NOTATIONS AND MAIN NOTIONS}

Let $\gamma: T \rightarrow \mathbb{R}^{2}$ be a continuous mapping of the scalar segment $T=(\hat{t}, \check{t}),-\infty \leqslant \hat{t}<\check{t} \leqslant+\infty$ into the plane. The vector function $\gamma(t)=\left(\gamma_{1}(t), \gamma_{2}(t)\right)$ is thrice continuously differentiable function everywhere on $T$ except for a finite set $T^{0} \subset T$ of points $t_{0} \in T^{0}$, at which the one-sided third order derivatives are finite and at least one the following identities is violated:

$$
\gamma_{1}^{\prime \prime \prime}\left(t_{0}-0\right)=\gamma_{1}^{\prime \prime \prime}\left(t_{0}+0\right), \quad \gamma_{2}^{\prime \prime \prime}\left(t_{0}-0\right)=\gamma_{2}^{\prime \prime \prime}\left(t_{0}+0\right) .
$$

The image $\Gamma=\{\mathbf{x}=\gamma(t): t \in T\}$ of this mapping is a planar curve. We include into consideration also contours, which are curves defined on finite intervals $T=(\hat{t}, \check{t}),-\infty<$ $\hat{t}<\check{t}<+\infty$, which can be defined at the end points $t=\hat{t}$ and $t=\check{t}$ so that $\gamma(\hat{t})=\gamma(\check{t})$.

In what follows we shall deal with local solutions of equations of form

$$
G\left(t_{1}, t_{2}\right)=0 \text {. }
$$

Here $G=G\left(t_{1}, t_{2}\right)$ is a symmetric function of two variables defined on the plane of the parameters $\left(t_{1}, t_{2}\right) \in \mathbb{R}^{2}$. The form of this function and its differential properties are determined in what follows. We seek the solutions of form $t_{2}=t_{2}\left(t_{1}\right)$ and $t_{1}=t_{1}\left(t_{2}\right)$ of this equation on rectangular open domains $\Pi_{+}\left(t_{0}\right)=\left\{\left(t_{1}, t_{2}\right) \in \mathbb{R}^{2}: t_{1} \in\left(t_{0}-\delta_{1}, t_{0}\right), t_{2} \in\left(t_{0}, t_{0}+\delta_{2}\right)\right\}$. Here $t_{0} \in T$ is fixed and $\delta_{1}>0, \delta_{2}>0$. As solutions of this equation, we mean local diffeomorphisms [17], [18]. In this case, discussing a local diffeomorphism, we suppose that it is defined in a half-neigbourhood (left or right one, depending on the situation) of a point under the consideration. A local diffeomorphism $t_{2}=t_{2}\left(t_{1}\right)$ defined by equation (3.1) is left semi-continuous at the point $t_{1}=t_{0}$ and maps the left half-neighbourhood of the point $t_{1}=t_{0}$ into its right hafl-neighbourhood if the following conditions are satisfied:

(A1) $t_{2}\left(\left(t_{0}-\delta_{1}, t_{0}\right)\right)=\left(t_{0}, t_{0}+\delta_{2}\right), \delta_{1}>0, \delta_{2}>0$, 
(A2) $\lim _{t_{1} \rightarrow t_{0}-0} t_{2}\left(t_{1}\right)=t_{0}$.

The local diffeomorphism $t_{1}=t_{1}\left(t_{2}\right)$ defined by equation (3.1) is right semi-continuous at the point $t_{1}=t_{0}$ and maps the right half-neighbourhood of the point $t_{2}=t_{0}$ into the right half-neighbourhood if the conditions similar to properties (A1), (A2) hold:

( $\underline{\text { A1 }) ~} t_{1}\left(\left(t_{0}, t_{0}+\delta_{2}\right)\right)=\left(t_{0}-\delta_{1}, t_{0}\right), \delta_{1}>0, \delta_{2}>0$

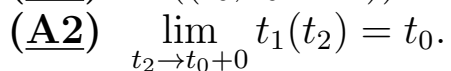

We note that Conditions (A2) and ( $\underline{\mathrm{A} 2})$, apart of the semi-continuity, expresses one of the ways of determining, in a limiting form, of fixed points. Here we mean the points $\left(t_{2}, t_{1}\right)=\left(t_{0}, t_{0}\right)$ lying on the bisectrix of the first and third coordinate angles in the space of the parameters, which are limiting for the graphs of the local diffeomorphisms. The existence of such points and corresponding local diffeomorphisms was shown at examples in [12].

We arbitrary choose and fix two arbitrary momenta $t_{1} \in T$ and $t_{2} \in T, t_{1}<t_{2}$. At the points $\gamma\left(t_{1}\right)$ and $\gamma\left(t_{2}\right)$ we draw tangential straight lines.

Definition 3.1. A pseudo-vertex of a curve $\Gamma$ is a point

$$
\mathbf{x}^{(0)}=\left(\gamma_{1}\left(t_{0}\right), \gamma_{2}\left(t_{0}\right)\right) \triangleq \lim _{t_{1} \rightarrow t_{0}-0}\left(x_{1}^{*}, x_{2}^{*}\right),
$$

where $\left(x_{1}^{*}, x_{2}^{*}\right)=\left(x_{1}^{*}\left(t_{1}, t_{2}\left(t_{1}\right)\right), x_{2}^{*}\left(t_{1}, t_{2}\left(t_{1}\right)\right)\right)$ is a one-parametric subset of solutions to the system of equations

$$
\left\{\begin{array}{l}
\left(x_{1}^{*}-\gamma_{1}\left(t_{1}\right)\right) \gamma_{2}^{\prime}\left(t_{1}\right)=\left(x_{2}^{*}-\gamma_{2}\left(t_{1}\right)\right) \gamma_{1}^{\prime}\left(t_{1}\right), \\
\left(x_{1}^{*}-\gamma_{1}\left(t_{2}\right)\right) \gamma_{2}^{\prime}\left(t_{2}\right)=\left(x_{2}^{*}-\gamma_{2}\left(t_{2}\right)\right) \gamma_{1}^{\prime}\left(t_{2}\right),
\end{array}\right.
$$

defined by a left-continuous at the point $t_{1}=t_{0}$ local diffeomorphism $t_{2}=t_{2}\left(t_{1}\right)$ of the left half-neighbourhood of the point $t_{1}=t_{0}$ into its right half-neighbourhood, which is defined by the equation

$$
G\left(t_{1}, t_{2}\right) \triangleq \rho^{2}\left(\gamma\left(t_{1}\right),\left(x_{1}^{*}, x_{2}^{*}\right)\right)-\rho^{2}\left(\gamma\left(t_{2}\right),\left(x_{1}^{*}, x_{2}^{*}\right)\right)=0 .
$$

From the point of view of geometry, $\left(x_{1}^{*}, x_{2}^{*}\right)$ is the point of intersection of the tangential lines to the curve $\Gamma$ at the points $\gamma\left(t_{1}\right)$ and $\gamma\left(t_{2}\right), G=G\left(t_{1}, t_{2}\right)$ is the difference of the squares of distances between the mentioned points $\gamma\left(t_{1}\right)$ and $\gamma\left(t_{2}\right)$ of the curve $\Gamma$ and the point $\left(x_{*}, x_{*}\right)$ of the intersections of the tangential lines drawn at $\gamma\left(t_{1}\right)$ and $\gamma\left(t_{2}\right)$.

While constructing the minimax solution to problem 2.1 or the corresponding fundamental solution, differing just by the sign, to problem (2.3) of the geometic optics with a constant refraction ratio, it is convenient to use some constructions of the geometric approximation theory. Here we the operator $P_{M}(\mathbf{x})$ of projecting points $\mathbf{x} \in \mathbb{R}^{2} \backslash M$ into $M$. It is known [19] that the solution to the eikonal equation is smooth in the entire domain in the case of single-valued $P_{M}(\mathbf{x}), \mathbf{x} \in \mathbb{R}^{2} \backslash M$, that is, as card $P_{M}(\mathbf{x})=1$. Such situation in the considered problems occurs in the case of a convex boundary set $M$. Then, according to the introduced terminology [20], the set $M$ is a "sun". In the general case when $M$ is a non-convex set, the set $M$ does not possess the sun property, since the number of the projections of the points in its complement can differ from one; here card $P_{M}(\mathbf{x})>1$.

Definition 3.2. A bisectrix of a set $M \subset \mathbb{R}^{2}$ is

$$
L=\left\{\mathbf{x} \in \mathbb{R}^{2} \backslash M: \operatorname{card} P_{M}(\mathbf{x})>1\right\} .
$$

Being applied to speed control problem with dynamics $(2.2)$, the set $L$ consists of the points, from which two or more optimal trajectories leave. Thus, the bisectrix $L$ of the set $M \subset \mathbb{R}^{2}$ is a singular set of the function of optimal result. It should be mentioned that the bisectrix is a set of symmetry, the topological properties of which are studied in the theory of singularities of smooth mappings, see, for instance, 21]. 
Definition 3.3. A branch $L\left(\mathbf{x}^{(0)}\right)$ of the bisectrix $L$ of a curve $\Gamma$, where $\mathbf{x}^{(0)}$ is a pseudovertex $\Gamma$ is called the set of points $\left(x_{1}, x_{2}\right) \in \mathbb{R}^{2}$ satisfying the system of equations

$$
\left\{\begin{array}{l}
\left(x_{1}-\gamma_{1}\left(t_{1}\right)\right) \gamma_{1}^{\prime}\left(t_{1}\right)+\left(x_{2}-\gamma_{2}\left(t_{1}\right)\right) \gamma_{2}^{\prime}\left(t_{1}\right)=0, \\
\left(x_{1}-\gamma_{1}\left(t_{2}\right)\right) \gamma_{1}^{\prime}\left(t_{2}\right)+\left(x_{2}-\gamma_{2}\left(t_{2}\right)\right) \gamma_{2}^{\prime}\left(t_{2}\right)=0,
\end{array}\right.
$$

where $t_{2}=t_{2}\left(t_{1}\right)$ is a left-continuous at the point $t_{1}=t_{0}$ local diffeomorphism of the left haflneighbourhood of the point $t_{1}=t_{0}$ into its right half-neighbourhood, which is defined by equation (3.3) and satisfies Conditions (A1), (A2).

By construction, system $(3.4)$ is dual to system $(3.2)$. Its solutions are points $\mathbf{x} \in \mathbb{R}^{2} \backslash \Gamma$ having two closest points on $M \subset \mathbb{R}^{2}$.

The pseudo-vertices and the branches of the bisectrix are main structural elements in constructing, analytic or combined numerical-analytic, of the singular set of problem (2.1).

Remark 3.1. The above definitions of the pseudo-vertex and the branch of the bisectrix are given by means of a local diffeomorphism of form $t_{2}=t_{2}\left(t_{1}\right)$. Taking into consideration of a symmetry of the mathematical model, definitions 3.1 and 3.3 can be reformulated in terms of local diffeomorphisms of form $t_{1}=t_{1}\left(t_{2}\right)$.

Let us introduce scalar characteristics of pseudo-vertices.

Definition 3.4. The left one-sided derivative

$$
\lambda \triangleq t_{2}^{\prime}\left(t_{0}-0\right)=\lim _{t_{1} \rightarrow t_{0}-0} \frac{t_{2}\left(t_{1}\right)-t_{0}}{t_{1}-t_{0}}
$$

is a left marker of a pseudo-vertex $\mathbf{x}^{(0)} \in \Gamma$; here $t_{2}=t_{2}\left(t_{1}\right)$ satisfies (A1), (A2).

Definition 3.5. The right one-sided derivative

$$
\mu \triangleq t_{1}^{\prime}\left(t_{0}+0\right)=\lim _{t_{2} \rightarrow t_{0}+0} \frac{t_{1}\left(t_{2}\right)-t_{0}}{t_{2}-t_{0}}
$$

is called a right marker of a pseudo-vertex $\mathbf{x}^{(0)} \in \Gamma$; here $t_{1}=t_{1}\left(t_{2}\right)$ satisfies $(\underline{A 1})$, (므).

An important implication of the symmetry of equation $(3.3)$ is a connection between onesided markers. If a local diffeomorphism $t_{2}=t_{2}\left(t_{1}\right), t_{1} \in\left(t_{0}-\delta_{1}, t_{0}\right)$, is a solution of (3.3), then the inverse local diffeomorphism $t_{1}=t_{1}\left(t_{2}\right), t_{2} \in\left(t_{0}, t_{0}+\delta_{2}\right)$, is also a solution of equation (3.3), see [10]. At that, due to semi-continuity conditions (A2) and (A2), their graphs have a common limiting point $\left(t_{1}, t_{2}\right)=\left(t_{0}, t_{0}\right)$. In this the one-sided markers are mutually reciprocal:

$$
\mu=\lambda^{-1} \text {. }
$$

We also note that $\lambda \leqslant 0$ since $t_{2}^{\prime}\left(t_{1}\right)<0, t_{1} \in\left(t_{0}-\delta_{1}, t_{0}\right), \delta_{1}>0$. Then by (3.7) we also have $\mu \leqslant 0$.

The problem on finding the conditions ensuring the existence of local solution of equation (3.3) satisfying conditions (A1), (A2) and ( $\underline{\mathrm{A} 1}),(\underline{\mathrm{A} 2})$, is an independent issue. The developing of the approaches for solving it is not restricted by the methods of classical analysis. Here a rather essential feature of the problem is the multiplicity of solutions contracting to the points in the graph of the identity function $t_{2}=t_{1}$. This function is an obvious solution of equation (3.3), but nevertheless, it does not fit the conditions on solutions to boundary value problems (2.1) and (2.3). One of the ways of finding out the properties of local diffeomorphisms satisfying conditions (A1), (A2) and ( $\underline{\mathrm{A} 1}),(\underline{\mathrm{A}} 2)$ is related with using transversality conditions [10]. We should also pay an attention to a connection (see conditions (A2) and (A2)) between the problem on existence of local diffeomorphism with the problem on existence of the fixed point in the space of parameters. The fixed point method has very wide applications in the analysis and the theory of differential equations. It is also applicable in studying non-smooth 
optimal control problems and in finding equilibrium states in game problems, see, for instance, 22 .

The markers of a pseudo-vertex fix a qualitative state of the curve from the point of view of differentiability. In other words, the values of one-sided markers indicate the smoothness order of the curve at the pseudo-vertex. For instance, in the case of a thrice differentiability of the curve at the pseudo-vertex its one-sided markers are equal [10]:

$$
\lambda=\mu=-1 .
$$

If the curve is smooth but does not have a classical curvature, then the one-sided markers at the pseudo-vertex take extremal values in its spectrum $[-\infty, 0]$, here $\lambda=0$ or $\lambda=-\infty$. Finally, if the first order derivatives are discontinuous, then the left marker is equal to the quotient of the differentials of the left and right arcs of the curve contracted to a point, for more details see [10]-15]. Respectively, here the right marker is equal to their reciprocal quotient.

Let us clarify the differential properties of the curve $\Gamma$ enveloping the boundary set $M$. We denote by $\operatorname{det}(\mathbf{a}, \mathbf{b})$ the second order determinant constructed by the vectors $\mathbf{a}=\left(a_{1}, a_{2}\right)$, $\mathbf{b}=\left(b_{1}, b_{2}\right)$ written as rows. We denote the scalar product of these vectors by $\langle\mathbf{a}, \mathbf{b}\rangle$. We shall assume that the following condition for the curve $\Gamma$ holds:

(B1) $\gamma^{\prime}(t) \neq(0,0), \quad t \in T$;

(B2) $\operatorname{det}\left(\gamma^{\prime}(t), \gamma^{\prime \prime}(t)\right) \neq 0, \quad t \in T^{0}$.

Condition (B1) means a regularity of the curve $\Gamma$. Condition (B2) yields that the curvature is non-zero and this ensures the existence of solutions to system (3.2). Moreover, these conditions considered in the system ensure that the coordinate functions of the curve are not planar at $t \in T^{0}$ [18]. This allows to employ the jet technique in approximative calculations [17], [23].

We denote the set of curves $\Gamma$ with the mentioned differential properties and obeying conditions (B1), (B2) by $\{\Gamma\}_{T}$.

In what follows we concentrate on finding out the properties of local solutions to equation (3.3) of form $t_{2}=t_{2}\left(t_{1}\right), t_{1} \in\left(t_{0}-\delta_{1}, t_{0}\right.$, in particular, on finding the value of the left marker. Earlier it was shown that a local diffeomorphism $t_{2}=t_{2}\left(t_{1}\right)$ satisfying conditions (A1), (A2) defines pseudo-vertex $\mathbf{x}^{(0)}=\mathbf{x}\left(t_{0}\right), t_{0} \in T$, of the curve $\Gamma$ and at the same time is a local solution to one of the branches of harmonic ratio type equation with two-parametric coefficients, see [13, Eq. (3.5)]. This equation arises as a reduction of main equation (3.3) and reads as 13 .

$$
G\left(t_{1}, t_{2}\right) \triangleq \frac{\gamma_{2}\left(t_{2}\right)-\gamma_{2}\left(t_{1}\right)}{\gamma_{1}\left(t_{2}\right)-\gamma_{1}\left(t_{1}\right)}-\frac{-\gamma_{1}^{\prime}\left(t_{1}\right) \gamma_{1}^{\prime}\left(t_{2}\right)+\gamma_{2}^{\prime}\left(t_{1}\right) \gamma_{2}^{\prime}\left(t_{2}\right)+s\left(t_{1}\right) s\left(t_{2}\right)}{\gamma_{2}^{\prime}\left(t_{1}\right) \gamma_{1}^{\prime}\left(t_{2}\right)+\gamma_{2}^{\prime}\left(t_{2}\right) \gamma_{1}^{\prime}\left(t_{1}\right)}=0
$$

Here $s(t)=\sqrt{\left(\gamma_{1}^{\prime}(t)\right)^{2}+\left(\gamma_{2}^{\prime}(t)\right)^{2}}, t \in T$. In analysis of the properties of solutions to these equation we employ the technique of jets basing on local expansions of scalar functions in the vicinity of the point $t=t_{0}$ by the Taylor formula. Following [17], by $k$-jet, where $k$ is a natural number, of a sufficiently many times differentiable function $f(t), t \in T$, we mean the Taylor polynomial of $k$ th order:

$$
J_{t}^{k} f\left(t_{0}\right)=\sum_{i=0}^{k} \frac{f^{(i)}\left(t_{0}\right)}{i !}\left(t-t_{0}\right)^{i} .
$$

Let $f(t)$ and $g(t)$ be differentiable functions. The sum of $k$-jets of these functions is found as the sum of the corresponding Taylor polynomials of degree $k$. The product of $k$-jets is defined by the following rule. If

$$
J_{t}^{k} f\left(t_{0}\right)=\sum_{i=0}^{k} \frac{f^{(i)}\left(t_{0}\right)}{i !}\left(t-t_{0}\right)^{i}, \quad J_{t}^{k} g\left(t_{0}\right)=\sum_{i=0}^{k} \frac{g^{(i)}\left(t_{0}\right)}{i !}\left(t-t_{0}\right)^{i}
$$


are $k$-jets of functions $f(t)$ and $g(t)$ respectively, then their product

$$
J_{t}^{k} f\left(t_{0}\right) \otimes J_{t}^{k} g\left(t_{0}\right)
$$

is a polynomial obtained by a natural term-by-term multiplication of the polynomials $J_{t}^{k} f\left(t_{0}\right)$ and $J_{t}^{k} g\left(t_{0}\right)$ in which all terms of degree exceeding $k$ are neglected.

In what follows we deal with 1-, 2- and 3-jets. Then the formed model is three-point with an imposed order relation. Here the point $t_{0} \in T$ is a central node and, as a rule, is fixed, while the points $t_{1} \in T$ and $t_{2} \in T$ are variables and $t_{1}<t_{0}<t_{2}$. The restrictions for the ordering of the points $t_{1}, t_{0}, t_{2}$ are due to the features of the singular curves for the eikonal equation, which are mentioned in classical work 24. Here singular lines are are scattering curves. Optimal motions of characteristic system (2.2) with an initial point at the scattering curve "roll down" from this curve in different sides. In the considered work the arguments of the end-points of these trajectories, which are segments here, are $t_{1} \in\left(t_{0}-\delta_{1}, t_{0}\right), \delta_{1}>0$ and $t_{2}=t_{2}\left(t_{1}\right), t_{2} \in\left(t_{0}, t_{0}+\delta_{1}\right), \delta_{2}>0$, where $t_{2}=t_{2}\left(t_{1}\right)$ is a local solution of equation (3.3) with properties (A1), (A2), since here $t_{1}<t_{0}<t_{2}$ and $t_{0}$ is the argument of the pseudo-vertex of the boundary of the boundary set.

We introduce the following notations for the increments:

$$
\Delta_{1}=t_{0}-t_{1}>0, \quad \Delta_{2}=t_{2}-t_{0}>0, \quad \Delta=\Delta_{1}+\Delta_{2} .
$$

We shall distinguish the jets obtained by the right differentiation, that is, as $t_{0}<t_{2}$ and the jest obtained by the left differentiation, that is, as $t_{0}>t_{1}$. Then two 2-jets differing by the direction of the differentiation read as

$$
\begin{aligned}
& J_{t_{2}}^{2} f\left(t_{0}\right) \triangleq J_{t_{0}+\Delta_{2}}^{2} f\left(t_{0}\right)=f\left(t_{0}\right)+\Delta_{2} f^{\prime}\left(t_{0}\right)+\frac{\Delta_{2}^{2}}{2} f^{\prime \prime}\left(t_{0}\right), \\
& J_{t_{1}}^{2} f\left(t_{0}\right) \triangleq J_{t_{0}-\Delta_{1}}^{2} f\left(t_{0}\right)=f\left(t_{0}\right)-\Delta_{1} f^{\prime}\left(t_{0}\right)+\frac{\Delta_{1}^{2}}{2} f^{\prime \prime}\left(t_{0}\right) .
\end{aligned}
$$

By the above given definition, the product of these 2-jets is a second order polynomial with respect to the increments $\Delta_{1}$ and $\Delta_{2}$, which are treated as independent variables:

$$
\begin{aligned}
J_{t_{2}}^{2} f\left(t_{0}\right) \otimes J_{t_{1}}^{2} g\left(t_{0}\right)= & \left(f\left(t_{0}\right)+\Delta_{2} f^{\prime}\left(t_{0}\right)+\frac{\Delta_{2}^{2}}{2} f^{\prime \prime}\left(t_{0}\right)\right) \otimes\left(g\left(t_{0}\right)-\Delta_{1} g^{\prime}\left(t_{0}\right)+\frac{\Delta_{1}^{2}}{2} g^{\prime \prime}\left(t_{0}\right)\right) \\
= & f\left(t_{0}\right) g\left(t_{0}\right)-\Delta_{1} f\left(t_{0}\right) g^{\prime}\left(t_{0}\right)+\Delta_{2} f^{\prime}\left(t_{0}\right) g\left(t_{0}\right)-\Delta_{1} \Delta_{2} f^{\prime}\left(t_{0}\right) g^{\prime}\left(t_{0}\right) \\
& +\frac{\Delta_{2}^{2}}{2} f^{\prime \prime}\left(t_{0}\right) g\left(t_{0}\right)+\frac{\Delta_{1}^{2}}{2} f\left(t_{0}\right) g^{\prime \prime}\left(t_{0}\right) .
\end{aligned}
$$

If it is necessary, we can govern the coefficients of the jets by introducing a dependence between the increments $\Delta_{1}$ and $\Delta_{2}$, for instance, by means of a local diffeomorphism. This approach is used in justifying the statements.

\section{MAIN RESUlT}

We consider the case of a non-stationary pseudo-vertex of the boundary of the boundary set, at which the smoothness of the curvature is broken. Let us justify the necessary conditions for the existence of a pseudo-vertex as generalized stationary conditions for the function $G\left(t_{1}, t_{2}\right)$. We shall also obtain algebraic equations for finding one-sided markers. We shall consider in details a procedure of calculating just one of two markers, namely, of the one-sided left marker.

Theorem 4.1. Let $\mathbf{x}^{(0)}=\left(\gamma_{1}\left(t_{0}\right), \gamma_{2}\left(t_{0}\right)\right)$ be a pseudo-vertex of the curve

$$
\Gamma=\{\mathbf{x}=\gamma(t): t \in T\} \in\{\Gamma\}_{T}
$$

in the boundary condition of Dirichlet problem (2.1), and $\mathbf{x}^{(0)}$ is defined by the local diffeomorphism $t_{2}=t_{2}\left(t_{1}\right)$ in (3.8) and conditions (A1), (A2) are satisfied. Assume that there exists a 
left marker $\lambda=t_{2}^{\prime}\left(t_{0}-0\right) \leqslant 0$, and the coordinate functions of the pseudo-vertex satisfy the non-stationarity conditions:

$$
\gamma_{1}^{\prime}\left(t_{0}\right) \neq 0, \quad \gamma_{2}^{\prime}\left(t_{0}\right) \neq 0
$$

and

$$
\gamma_{i}^{\prime \prime \prime}\left(t_{0}-0\right) \neq \gamma_{i}^{\prime \prime \prime}\left(t_{0}+0\right)
$$

for at least one $i \in\{1,2\}$. Then the following identities necessary hold:

$$
\begin{gathered}
\lim _{t_{1} \rightarrow t_{0}-0} \frac{\partial G\left(t_{1}, t_{2}\left(t_{1}\right)\right)}{\partial t_{1}}=0, \quad \lim _{t_{1} \rightarrow t_{0}-0} \frac{\partial G\left(t_{1}, t_{2}\left(t_{1}\right)\right)}{\partial t_{2}}=0, \\
\lambda^{2}(\lambda-3) \operatorname{det}\left(\gamma^{\prime}\left(t_{0}\right), \gamma_{+}^{\prime \prime \prime}\left(t_{0}\right)\right)+(3 \lambda-1) \operatorname{det}\left(\gamma^{\prime}\left(t_{0}\right), \gamma_{-}^{\prime \prime \prime}\left(t_{0}\right)\right) \\
=\frac{3 \operatorname{det}\left(\gamma^{\prime}\left(t_{0}\right), \gamma^{\prime \prime}\left(t_{0}\right)\right)\left\langle\gamma^{\prime}\left(t_{0}\right), \gamma^{\prime \prime}\left(t_{0}\right)\right\rangle}{s^{2}}(\lambda-1)^{3} .
\end{gathered}
$$

Proof. It follows from (3.8) that at the points $\left(t_{1}, t_{2}\right)=\left(t_{1}, t_{2}\left(t_{1}\right)\right)$ of the graph of the local diffeomorphism the identity holds:

$$
\frac{\partial G}{\partial t_{1}}+t_{2}^{\prime} \frac{\partial G}{\partial t_{2}}=0
$$

We pass to the limit along the local diffeomorphism $t_{2}=t_{2}\left(t_{1}\right)$ (see also [10]):

$$
\lim _{t_{1} \rightarrow t_{0}-0}\left(\frac{\partial Q\left(t_{1}, t_{2}\left(t_{1}\right)\right)}{\partial t_{1}}+t_{2}^{\prime} \frac{\partial Q\left(t_{1}, t_{2}\left(t_{1}\right)\right)}{\partial t_{2}}\right)=0 .
$$

Identity 4.5 expresses the condition of transversal (puncturing) intersection of the closure of the graph of the local diffeomorphism $t_{2}=t_{2}\left(t_{1}\right)$ with the graph of the identity diffeomorphism $t_{2}=t_{1}$ at the common limiting point $\left(t_{1}, t_{2}\right)=\left(t_{0}, t_{0}\right)$.

Basing on the transversality condition, let us find out a series of properties of the pseudovertex. By assumptions of Theorem 4.1. the left marker exists and finite and (4.5) implies the formula for this marker:

$$
t_{2}^{\prime}\left(t_{0}-0\right)=-\lim _{t_{1} \rightarrow t_{0}-0}\left(\frac{\partial Q\left(t_{1}, t_{2}\left(t_{1}\right)\right)}{\partial t_{1}} \cdot\left(\frac{\partial Q\left(t_{1}, t_{2}\left(t_{1}\right)\right)}{\partial t_{2}}\right)^{-1}\right) .
$$

Now we are going to find the approximations for partial derivatives considered along the diffeomorphism $t_{2}=t_{2}\left(t_{1}\right)$. We first adopt a series of conventions aimed on reducing the calculations. In all approximation constructions, the point of the expansion is fixed, and $t_{2}=t_{0}$ and $t_{1}=t_{0}$ depending on the direction of the expansion, to right or to the left. For the sake of brevity, the value $t_{0}$ of the variables in $t_{2}$ and $t_{1}$ is omitted. Our lines of calculations first suggest to find approximations for arbitrary positive increments $\Delta_{1}=t_{0}-t_{1}, \Delta_{2}=t_{2}-t_{0}$ and we approximate for the increments related due to the local diffeomorphism $t_{2}=t_{2}\left(t_{1}\right)$. It is important to note that as the triple of the points is related by the local diffeomorphism $t_{2}=t_{2}\left(t_{1}\right)$, the increment $\Delta_{2}=\Delta_{2}\left(\Delta_{1}\right)=t_{2}\left(t_{1}\right)-t_{0}$, depends on $\Delta_{1}$ and

$$
\Delta_{2}=\Delta_{2}\left(\Delta_{1}\right)=t_{2}\left(t_{1}\right)-t_{0}=t_{2}^{\prime}\left(t_{0}-0\right)\left(t_{1}-t_{0}\right)+o\left(t_{1}-t_{0}\right)=-\lambda \Delta_{1}+o\left(\Delta_{1}\right) .
$$

In particular, this implies

$$
\Delta=\Delta_{1}+\Delta_{2}\left(\Delta_{1}\right)=\Delta_{1}-\lambda \Delta_{1}+o\left(\Delta_{1}\right)=(1-\lambda) \Delta_{1}+o\left(\Delta_{1}\right), \quad \text { where } 1-\lambda \neq 0 .
$$

Hereinafter the notations of form $o\left(\Delta_{1}^{k}\right)$, where $k=1,2,3$, are used for the functions having a higher smallness order with respect to the argument to the left of the consideration point, that is,

$$
\lim _{\Delta_{1} \downarrow 0} \frac{o\left(\Delta_{1}^{k}\right)}{\Delta_{1}^{k}}=0
$$


The notation $\varepsilon\left(\Delta_{1}\right)$ is used for the functions infinitesimal in the left half-neighbourhood of the consideration point; here

$$
\lim _{\Delta_{1} \downarrow 0} \varepsilon\left(\Delta_{1}\right)=0
$$

We also define that two scalar functions $y=q(t)$ and $y=g(t)$ are equivalent to the left of the consideration point $t=t_{0} \in \mathbb{R}$ if

$$
\lim _{t_{1} \rightarrow t_{0}-0} \frac{q(t)}{g(t)}=1
$$

In this case we write $q(t) \sim g(t), t_{1} \rightarrow t_{0}-0$.

For the coordinate functions $\gamma_{1}(t), \gamma_{2}(t)$ and for their derivatives calculated at the central node $t_{0}$ as well as for the jets corresponding to the coordinate functions, we do not write the variable. For one-sided derivatives we change the notations $t_{0}-0$ and $t_{0}+0$ by moving the sign minus or plus to the subscript:

$$
\gamma_{i}^{\prime}=\gamma_{i}^{\prime}\left(t_{0}\right), \quad \gamma_{i}^{\prime \prime}=\gamma_{i}^{\prime \prime}\left(t_{0}\right), \quad \gamma_{i,-}^{\prime \prime \prime}=\gamma_{i}^{\prime \prime \prime}\left(t_{0}-0\right), \quad \gamma_{i,+}^{\prime \prime \prime}=\gamma_{i}^{\prime \prime \prime}\left(t_{0}+0\right), \quad i=1,2 .
$$

We begin with the expansion for the partial derivative with respect to the first variable. We have:

$$
\begin{aligned}
\frac{\partial G\left(t_{1}, t_{2}\right)}{\partial t_{1}}= & \frac{\operatorname{det}\left(\gamma^{\prime}\left(t_{1}\right), \gamma\left(t_{2}\right)-\gamma\left(t_{1}\right)\right)}{\left(\gamma_{1}\left(t_{2}\right)-\gamma_{1}\left(t_{1}\right)\right)^{2}}-\frac{s^{2}\left(t_{2}\right) \operatorname{det}\left(\gamma^{\prime}\left(t_{1}\right), \gamma^{\prime \prime}\left(t_{1}\right)\right)}{\left(\gamma_{2}^{\prime}\left(t_{1}\right) \gamma_{1}^{\prime}\left(t_{2}\right)+\gamma_{2}^{\prime}\left(t_{2}\right) \gamma_{1}^{\prime}\left(t_{1}\right)\right)^{2}} \\
& +\frac{s\left(t_{2}\right)\left[s\left(t_{1}\right)\left(\gamma_{2}^{\prime \prime}\left(t_{1}\right) \gamma_{1}^{\prime}\left(t_{2}\right)+\gamma_{2}^{\prime}\left(t_{2}\right) \gamma_{1}^{\prime \prime}\left(t_{1}\right)\right)-s^{\prime}\left(t_{1}\right)\left(\gamma_{2}^{\prime}\left(t_{1}\right) \gamma_{1}^{\prime}\left(t_{2}\right)+\gamma_{2}^{\prime}\left(t_{2}\right) \gamma_{1}^{\prime}\left(t_{1}\right)\right)\right]}{\left(\gamma_{2}^{\prime}\left(t_{1}\right) \gamma_{1}^{\prime}\left(t_{2}\right)+\gamma_{2}^{\prime}\left(t_{2}\right) \gamma_{1}^{\prime}\left(t_{1}\right)\right)^{2}}
\end{aligned}
$$

We introduce the notations:

$$
\frac{\partial G\left(t_{1}, t_{2}\right)}{\partial t_{1}}=g_{1}\left(t_{1}, t_{2}\right)-g_{2}\left(t_{1}, t_{2}\right)+g_{3}\left(t_{1}, t_{2}\right)
$$

Here

$$
g_{1}\left(t_{1}, t_{2}\right)=\frac{g_{11}\left(t_{1}, t_{2}\right)}{g_{12}\left(t_{1}, t_{2}\right)}, \quad g_{2}\left(t_{1}, t_{2}\right)=\frac{g_{21}\left(t_{1}, t_{2}\right)}{g_{22}\left(t_{1}, t_{2}\right)}, \quad g_{3}\left(t_{1}, t_{2}\right)=\frac{g_{31}\left(t_{1}, t_{2}\right)}{g_{32}\left(t_{1}, t_{2}\right)}
$$

are corresponding quotients in the expansion.

Let us find the approximations for the selected quotients applying, where it is appropriate, the technique of jets. We begin with approximating the numerator of the first quotient of order $o\left(\Delta_{12}^{3}\right)$, where $\Delta_{12}=\max \left\{\Delta_{1}, \Delta_{2}\right\}$. Hereinafter, in view of the bulkiness of calculations, which are of course important but technical, we omit a series of intermediate calculations, which can be recovered by an interesting reader.

Let us write the expansions for the coordinate functions constructed at the points located in different sides of the consideration point $t_{0} \in \mathbb{R}$ :

$$
\begin{aligned}
& \gamma_{i}\left(t_{2}\right)=\gamma_{i}+\Delta_{2} \gamma_{i}^{\prime}+\frac{\Delta_{2}^{2}}{2} \gamma_{i}^{\prime \prime}+\frac{\Delta_{2}^{3}}{6} \gamma_{i,+}^{\prime \prime \prime}+o\left(\Delta_{2}^{3}\right) \\
& \gamma_{i}\left(t_{1}\right)=\gamma_{i}-\Delta_{1} \gamma_{i}^{\prime}+\frac{\Delta_{1}^{2}}{2} \gamma_{i}^{\prime \prime}-\frac{\Delta_{1}^{3}}{6} \gamma_{i,-}^{\prime \prime \prime}+o\left(\Delta_{1}^{3}\right), \quad i=1,2
\end{aligned}
$$


Then

$$
\begin{aligned}
& g_{11}\left(t_{1}, t_{2}\right)=\operatorname{det}\left(\gamma^{\prime}\left(t_{1}\right), \gamma\left(t_{2}\right)-\gamma\left(t_{1}\right)\right) \\
& =\operatorname{det}\left(\gamma^{\prime}-\Delta_{1} \gamma^{\prime \prime}+\frac{\Delta_{1}^{2}}{2} \gamma_{-}^{\prime \prime \prime}+o\left(\Delta_{1}^{2}\right), \gamma+\Delta_{2} \gamma^{\prime}+\frac{\Delta_{2}^{2}}{2} \gamma^{\prime \prime}+\frac{\Delta_{2}^{3}}{6} \gamma_{+}^{\prime \prime \prime}+o\left(\Delta_{2}^{3}\right) \gamma^{\prime}\right. \\
& \left.-\gamma_{+} \Delta_{1}-\frac{\Delta_{1}^{2}}{2} \gamma^{\prime \prime}+\frac{\Delta_{1}^{3}}{6} \gamma_{-}^{\prime \prime \prime}+o\left(\Delta_{1}^{3}\right)\right) \\
& =\operatorname{det}\left(\gamma^{\prime}-\Delta_{1} \gamma^{\prime \prime}+\frac{\Delta_{1}^{2}}{2} \gamma_{-}^{\prime \prime \prime}+o\left(\Delta_{1}^{2}\right), \Delta \gamma^{\prime}+\Delta \cdot \frac{\Delta_{2}-\Delta_{1}}{2} \gamma^{\prime \prime}+\frac{\Delta_{2}^{3}}{6} \gamma_{+}^{\prime \prime \prime}\right. \\
& \left.+\frac{\Delta_{1}^{3}}{6} \gamma_{-}^{\prime \prime \prime}+o\left(\Delta_{12}^{3}\right)\right) \\
& =\Delta \operatorname{det}\left(\gamma^{\prime}-\Delta_{1} \gamma^{\prime \prime}+\frac{\Delta_{1}^{2}}{2} \gamma_{-}^{\prime \prime \prime}+o\left(\Delta_{1}^{2}\right), \gamma^{\prime}+\frac{\Delta_{2}-\Delta_{1}}{2} \cdot \gamma^{\prime \prime}+\frac{\Delta_{2}^{3}}{6 \Delta} \gamma_{+}^{\prime \prime \prime}\right. \\
& \left.+\frac{\Delta_{1}^{3}}{6 \Delta} \gamma_{-}^{\prime \prime \prime}+o\left(\Delta_{12}^{2}\right)\right) \\
& =\Delta\left(\operatorname{det}\left(\gamma^{\prime}, \gamma^{\prime}\right)+\frac{\Delta_{2}-\Delta_{1}}{2} \operatorname{det}\left(\gamma^{\prime}, \gamma^{\prime \prime}\right)+\frac{\Delta_{2}^{3}}{6 \Delta} \operatorname{det}\left(\gamma^{\prime}, \gamma_{+}^{\prime \prime \prime}\right)\right. \\
& +\frac{\Delta_{1}^{3}}{6 \Delta} \operatorname{det}\left(\gamma^{\prime}, \gamma_{-}^{\prime \prime \prime}\right)-\Delta_{1} \operatorname{det}\left(\gamma^{\prime \prime}, \gamma^{\prime}\right)-\Delta_{1} \frac{\Delta_{2}-\Delta_{1}}{2} \operatorname{det}\left(\gamma^{\prime \prime}, \gamma^{\prime \prime}\right) \\
& \left.+\frac{\Delta_{1}^{2}}{2} \operatorname{det}\left(\gamma_{-}^{\prime \prime \prime}, \gamma^{\prime}\right)+o\left(\Delta_{12}^{2}\right)\right) \\
& =\Delta\left(\frac{\Delta}{2} \operatorname{det}\left(\gamma^{\prime}, \gamma^{\prime \prime}\right)+\frac{\Delta_{2}^{3}}{6 \Delta} \operatorname{det}\left(\gamma^{\prime}, \gamma_{+}^{\prime \prime \prime}\right)+\left(\frac{\Delta_{1}^{3}}{6 \Delta}-\frac{\Delta_{1}^{2}}{2}\right) \operatorname{det}\left(\gamma^{\prime}, \gamma_{-}^{\prime \prime \prime}\right)+o\left(\Delta_{12}^{2}\right)\right) \\
& =\Delta^{2}\left(\frac{\operatorname{det}\left(\gamma^{\prime}, \gamma^{\prime \prime}\right)}{2}+\frac{\Delta_{2}^{3}}{6 \Delta^{2}} \operatorname{det}\left(\gamma^{\prime}, \gamma_{+}^{\prime \prime \prime}\right)+\frac{\Delta_{1}^{3}-3 \Delta \Delta_{1}^{2}}{6 \Delta^{2}} \operatorname{det}\left(\gamma^{\prime}, \gamma_{-}^{\prime \prime \prime}\right)+o\left(\Delta_{12}\right)\right) \text {. }
\end{aligned}
$$

We note that inequality (4.2) imposed for one-sided third order derivatives complicate essentially approximation constructions.

We approximate the numeration of the first quotient by means of 3-jets [13]:

$$
\begin{aligned}
g_{12}\left(t_{1}, t_{2}\right) & \approx\left(J_{t_{2}}^{3} \gamma_{1}-J_{t_{1}}^{3} \gamma_{1}\right)^{2} \\
& =\left(\Delta_{2} \gamma_{1}^{\prime}+\frac{\Delta_{2}^{2}}{2} \gamma_{1}^{\prime \prime}+\frac{\Delta_{2}^{3}}{6} \gamma_{1,+}^{\prime \prime \prime}+\Delta_{1} \gamma_{1}^{\prime}-\frac{\Delta_{1}^{2}}{2} \gamma_{1}^{\prime \prime}+\frac{\Delta_{1}^{3}}{6} \gamma_{1,-}^{\prime \prime \prime}\right)^{2} \\
& =\left(\Delta \gamma_{1}^{\prime}+\frac{\Delta_{2}-\Delta_{1}}{2} \Delta \gamma_{1}^{\prime \prime}+\frac{\Delta_{2}^{3}}{6} \gamma_{1,+}^{\prime \prime \prime}+\frac{\Delta_{1}^{3}}{6} \gamma_{1,-}^{\prime \prime \prime}\right)^{2} \\
& =\Delta^{2}\left(\gamma_{1}^{\prime}\right)^{2}+\Delta^{2}\left(\Delta_{2}-\Delta_{1}\right) \gamma_{1}^{\prime} \gamma_{1}^{\prime \prime}=\Delta^{2}\left(\left(\gamma_{1}^{\prime}\right)^{2}+\left(\Delta_{2}-\Delta_{1}\right) \gamma_{1}^{\prime} \gamma_{1}^{\prime \prime}\right) .
\end{aligned}
$$

Thus,

$$
g_{12}\left(t_{1}, t_{2}\right)=\Delta^{2}\left(\left(\gamma_{1}^{\prime}\right)^{2}+\left(\Delta_{2}-\Delta_{1}\right) \gamma_{1}^{\prime} \gamma_{1}^{\prime \prime}\right)+o\left(\Delta_{12}^{3}\right)
$$

We consider the first quotient $g_{1}\left(t_{1}, t_{2}\right)$ and in view of the cancellation of numerator (4.9) and denominator 4.10 in approximation expansions by $\Delta^{2}$ under the natural reducing of 
approximation orders by two we get:

$$
\begin{aligned}
g_{1}\left(t_{1}, t_{2}\right)= & \frac{\frac{1}{2} \operatorname{det}\left(\gamma^{\prime}, \gamma^{\prime \prime}\right)+\frac{\Delta_{2}^{3}}{6 \Delta^{2}} \operatorname{det}\left(\gamma^{\prime}, \gamma_{+}^{\prime \prime \prime}\right)}{\left(\gamma_{1}^{\prime}\right)^{2}+\left(\Delta_{2}-\Delta_{1}\right) \gamma_{1}^{\prime} \gamma_{1}^{\prime \prime}+o\left(\Delta_{12}\right)} \\
& +\frac{\frac{\Delta_{1}^{3}-3 \Delta \Delta_{1}^{2}}{6 \Delta^{2}} \operatorname{det}\left(\gamma^{\prime}, \gamma_{-}^{\prime \prime \prime}\right)+o\left(\Delta_{12}\right)}{\left(\gamma_{1}^{\prime}\right)^{2}+\left(\Delta_{2}-\Delta_{1}\right) \gamma_{1}^{\prime} \gamma_{1}^{\prime \prime}+o\left(\Delta_{12}\right)}, \quad \Delta_{12} \rightarrow 0 .
\end{aligned}
$$

We proceed to the second quotient $g_{2}\left(t_{1}, t_{2}\right)=\frac{g_{21}\left(t_{1}, t_{2}\right)}{g_{22}\left(t_{1}, t_{2}\right)}$. We approximate the denominator of the second quotient (and in view of the identity, also that of the third quotient) by means of 1-jets:

$$
\begin{aligned}
g_{22}\left(t_{1}, t_{2}\right) & \approx\left(J_{t_{1}}^{1} \gamma_{2}^{\prime} \otimes J_{t_{2}}^{1} \gamma_{1}^{\prime}+J_{t_{2}}^{1} \gamma_{2}^{\prime} \otimes J_{t_{1}}^{1} \gamma_{1}^{\prime}\right)^{2} \\
& =\left(\left(\gamma_{2}^{\prime}-\Delta_{1} \gamma_{2}^{\prime \prime}\right) \otimes\left(\gamma_{1}^{\prime}+\Delta_{2} \gamma_{1}^{\prime \prime}\right)+\left(\gamma_{2}^{\prime}+\Delta_{2} \gamma_{2}^{\prime \prime}\right) \otimes\left(\gamma_{1}^{\prime}-\Delta_{1} \gamma_{1}^{\prime \prime}\right)\right)^{2} \\
& =\left(\gamma_{1}^{\prime} \gamma_{2}^{\prime}+\Delta_{2} \gamma_{2}^{\prime} \gamma_{1}^{\prime \prime}-\Delta_{1} \gamma_{1}^{\prime} \gamma_{2}^{\prime \prime}+\gamma_{1}^{\prime} \gamma_{2}^{\prime}-\Delta_{1} \gamma_{2}^{\prime} \gamma_{1}^{\prime \prime}+\Delta_{2} \gamma_{1}^{\prime} \gamma_{2}^{\prime \prime}\right)^{2} \\
& =\left(2 \gamma_{1}^{\prime} \gamma_{2}^{\prime}+\left(\Delta_{2}-\Delta_{1}\right) \gamma_{2}^{\prime} \gamma_{1}^{\prime \prime}+\left(\Delta_{2}-\Delta_{1}\right) \gamma_{1}^{\prime} \gamma_{2}^{\prime \prime}\right)^{2} \\
& =\left(2 \gamma_{1}^{\prime} \gamma_{2}^{\prime}+\left(\Delta_{2}-\Delta_{1}\right)\left(\gamma_{2}^{\prime} \gamma_{1}^{\prime \prime}+\gamma_{1}^{\prime} \gamma_{2}^{\prime \prime}\right)\right)^{2} \\
& =\left(2 \gamma_{1}^{\prime} \gamma_{2}^{\prime}\right)^{2}+4\left(\Delta_{2}-\Delta_{1}\right) \gamma_{1}^{\prime} \gamma_{2}^{\prime}\left(\gamma_{2}^{\prime} \gamma_{1}^{\prime \prime}+\gamma_{1}^{\prime} \gamma_{2}^{\prime \prime}\right)
\end{aligned}
$$

Hence, the following expansion holds:

$$
\begin{aligned}
g_{22}\left(t_{1}, t_{2}\right) & =g_{32}\left(t_{1}, t_{2}\right) \\
& =\left(2 \gamma_{1}^{\prime} \gamma_{2}^{\prime}\right)^{2}+4\left(\Delta_{2}-\Delta_{1}\right) \gamma_{1}^{\prime} \gamma_{2}^{\prime}\left(\gamma_{2}^{\prime} \gamma_{1}^{\prime \prime}+\gamma_{1}^{\prime} \gamma_{2}^{\prime \prime}\right)+o\left(\Delta_{12}\right), \quad \Delta_{12} \rightarrow 0 .
\end{aligned}
$$

By means of 1-jets we approximate the numerator of the second quotient of order $o\left(\Delta_{12}\right)$ :

$$
\begin{aligned}
g_{21}\left(t_{1}, t_{2}\right) & \approx J_{t_{2}}^{1} s^{2} \otimes J_{t_{1}}^{1} \operatorname{det}\left(\gamma^{\prime}, \gamma^{\prime \prime}\right) \\
& =\left(s^{2}+2 \Delta_{2} s s^{\prime}\right) \otimes\left(\operatorname{det}\left(\gamma^{\prime}, \gamma^{\prime \prime}\right)-\Delta_{1}\left(\operatorname{det}\left(\gamma^{\prime \prime}, \gamma^{\prime \prime}\right)+\operatorname{det}\left(\gamma^{\prime}, \gamma_{-}^{\prime \prime \prime}\right)\right)\right) \\
& =s^{2} \operatorname{det}\left(\gamma^{\prime}, \gamma^{\prime \prime}\right)-\Delta_{1} s^{2} \operatorname{det}\left(\gamma^{\prime}, \gamma_{-}^{\prime \prime \prime}\right)+2 \Delta_{2} s s^{\prime} \operatorname{det}\left(\gamma^{\prime}, \gamma^{\prime \prime}\right) .
\end{aligned}
$$

Then

$$
\begin{aligned}
g_{21}\left(t_{1}, t_{2}\right)= & s^{2} \operatorname{det}\left(\gamma^{\prime}, \gamma^{\prime \prime}\right)-\Delta_{1} s^{2} \operatorname{det}\left(\gamma^{\prime}, \gamma_{-}^{\prime \prime \prime}\right) \\
& +2 \Delta_{2} s s^{\prime} \operatorname{det}\left(\gamma^{\prime}, \gamma^{\prime \prime}\right)+o\left(\Delta_{12}\right), \quad \Delta_{12} \rightarrow 0 .
\end{aligned}
$$

In view of 4.12 and 4.13 the second quotient is expanded as follows:

$$
\begin{aligned}
g_{2}\left(t_{1}, t_{2}\right)= & \frac{s^{2} \operatorname{det}\left(\gamma^{\prime}, \gamma^{\prime \prime}\right)-\Delta_{1} s^{2} \operatorname{det}\left(\gamma^{\prime}, \gamma_{-}^{\prime \prime \prime}\right)+}{\left(2 \gamma_{1}^{\prime} \gamma_{2}^{\prime}\right)^{2}+4\left(\Delta_{2}-\Delta_{1}\right) \gamma_{1}^{\prime} \gamma_{2}^{\prime}\left(\gamma_{2}^{\prime} \gamma_{1}^{\prime \prime}+\gamma_{1}^{\prime} \gamma_{2}^{\prime \prime}\right)+o\left(\Delta_{12}\right)} \\
& +\frac{+2 \Delta_{2} s s^{\prime} \operatorname{det}\left(\gamma^{\prime}, \gamma^{\prime \prime}\right)+o\left(\Delta_{12}\right)}{\left(2 \gamma_{1}^{\prime} \gamma_{2}^{\prime}\right)^{2}+4\left(\Delta_{2}-\Delta_{1}\right) \gamma_{1}^{\prime} \gamma_{2}^{\prime}\left(\gamma_{2}^{\prime} \gamma_{1}^{\prime \prime}+\gamma_{1}^{\prime} \gamma_{2}^{\prime \prime}\right)+o\left(\Delta_{12}\right)},
\end{aligned}
$$

By means of 1-jets we approximate the numerator of the third quotient:

$$
\begin{aligned}
& s\left(t_{2}\right)\left[s\left(t_{1}\right)\left(\gamma_{2}^{\prime \prime}\left(t_{1}\right) \gamma_{1}^{\prime}\left(t_{2}\right)+\gamma_{2}^{\prime}\left(t_{2}\right) \gamma_{1}^{\prime \prime}\left(t_{1}\right)\right)-s^{\prime}\left(t_{1}\right)\left(\gamma_{2}^{\prime}\left(t_{1}\right) \gamma_{1}^{\prime}\left(t_{2}\right)+\gamma_{2}^{\prime}\left(t_{2}\right) \gamma_{1}^{\prime}\left(t_{1}\right)\right)\right] \\
& \approx\left(s+\Delta_{2} s^{\prime}\right) \otimes\left(\gamma_{2}^{\prime \prime} \gamma_{1}^{\prime} s+\gamma_{2}^{\prime} \gamma_{1}^{\prime \prime} s-2 \gamma_{1}^{\prime} \gamma_{2}^{\prime} s^{\prime}+2 \Delta_{2} \gamma_{1}^{\prime \prime} \gamma_{2}^{\prime \prime} s-\Delta_{1} \gamma_{2,-}^{\prime \prime \prime} \gamma_{1}^{\prime} s-\Delta_{1} \gamma_{1,-}^{\prime \prime \prime} \gamma_{2}^{\prime} s\right. \\
& \left.-\Delta_{1} \gamma_{2}^{\prime \prime} \gamma_{1}^{\prime} s^{\prime}-\Delta_{1} \gamma_{2}^{\prime} \gamma_{1}^{\prime \prime} s^{\prime}-\Delta_{2} \gamma_{2}^{\prime} \gamma_{1}^{\prime \prime} s^{\prime}-\Delta_{2} \gamma_{1}^{\prime} \gamma_{2}^{\prime \prime} s^{\prime}+\Delta_{1} \gamma_{1}^{\prime} \gamma_{2}^{\prime \prime} s^{\prime}+\Delta_{1} \gamma_{2}^{\prime} \gamma_{1}^{\prime \prime} s^{\prime}+2 \Delta_{1} \gamma_{1}^{\prime} \gamma_{2}^{\prime} s_{-}^{\prime \prime}\right) \\
& =\gamma_{2}^{\prime \prime} \gamma_{1}^{\prime} s^{2}+\gamma_{2}^{\prime} \gamma_{1}^{\prime \prime} s^{2}-2 \gamma_{1}^{\prime} \gamma_{2}^{\prime} s^{\prime} s+\Delta_{2} \gamma_{2}^{\prime \prime} \gamma_{1}^{\prime} s s^{\prime}+\Delta_{2} \gamma_{2}^{\prime} \gamma_{1}^{\prime \prime} s s^{\prime}-2 \Delta_{2} \gamma_{1}^{\prime} \gamma_{2}^{\prime}\left(s^{\prime}\right)^{2} \\
& +2 \Delta_{2} \gamma_{1}^{\prime \prime} \gamma_{2}^{\prime \prime} s^{2}-\Delta_{1} \gamma_{2,-}^{\prime \prime \prime} \gamma_{1}^{\prime} s^{2}-\Delta_{1} \gamma_{1,-}^{\prime \prime \prime} \gamma_{2}^{\prime} s^{2}-\Delta_{1} \gamma_{2}^{\prime \prime} \gamma_{1}^{\prime} s s^{\prime}-\Delta_{1} \gamma_{2}^{\prime} \gamma_{1}^{\prime \prime} s s^{\prime} \\
& -\Delta_{2} \gamma_{2}^{\prime} \gamma_{1}^{\prime \prime} s s^{\prime}-\Delta_{2} \gamma_{1}^{\prime} \gamma_{2}^{\prime \prime} s s^{\prime}+\Delta_{1} \gamma_{1}^{\prime} \gamma_{2}^{\prime \prime} s s^{\prime}+\Delta_{1} \gamma_{2}^{\prime} \gamma_{1}^{\prime \prime} s s^{\prime}+2 \Delta_{1} \gamma_{1}^{\prime} \gamma_{2}^{\prime} s s_{-}^{\prime \prime} \\
& =\gamma_{2}^{\prime \prime} \gamma_{1}^{\prime} s^{2}+\gamma_{2}^{\prime} \gamma_{1}^{\prime \prime} s^{2}-2 \gamma_{1}^{\prime} \gamma_{2}^{\prime} s^{\prime} s-2 \Delta_{2} \gamma_{1}^{\prime} \gamma_{2}^{\prime}\left(s^{\prime}\right)^{2}+2 \Delta_{2} \gamma_{1}^{\prime \prime} \gamma_{2}^{\prime \prime} s^{2}
\end{aligned}
$$




$$
-\Delta_{1} \gamma_{2,-}^{\prime \prime \prime} \gamma_{1}^{\prime} s^{2}-\Delta_{1} \gamma_{1,-}^{\prime \prime \prime} \gamma_{2}^{\prime} s^{2}+2 \Delta_{1} \gamma_{1}^{\prime} \gamma_{2}^{\prime} s s_{-}^{\prime \prime} .
$$

Therefore,

$$
\begin{aligned}
g_{31}\left(t_{1}, t_{2}\right)= & s^{2} \gamma_{2}^{\prime \prime} \gamma_{1}^{\prime}+s^{2} \gamma_{2}^{\prime} \gamma_{1}^{\prime \prime}-2 s s^{\prime} \gamma_{2}^{\prime} \gamma_{1}^{\prime}-2 \Delta_{2}\left(s^{\prime}\right)^{2} \gamma_{2}^{\prime} \gamma_{1}^{\prime} \\
& +\Delta_{1}\left(-s^{2} \gamma_{2,-}^{\prime \prime \prime} \gamma_{1}^{\prime}-s^{2} \gamma_{1,-}^{\prime \prime \prime} \gamma_{2}^{\prime}+2 s s_{-}^{\prime \prime} \gamma_{2}^{\prime} \gamma_{1}^{\prime}\right)+2 \Delta_{2} s^{2} \gamma_{1}^{\prime \prime} \gamma_{2}^{\prime \prime}+o\left(\Delta_{12}\right), \quad \Delta_{12} \rightarrow 0
\end{aligned}
$$

The expansion of the third quotient reads as

$$
\begin{aligned}
g_{3}\left(t_{1}, t_{2}\right)= & \frac{s^{2} \gamma_{2}^{\prime \prime} \gamma_{1}^{\prime}+s^{2} \gamma_{2}^{\prime} \gamma_{1}^{\prime \prime}-2 s s^{\prime} \gamma_{2}^{\prime} \gamma_{1}^{\prime}-2 \Delta_{2}\left(s^{\prime}\right)^{2} \gamma_{2}^{\prime} \gamma_{1}^{\prime}}{\left(2 \gamma_{1}^{\prime} \gamma_{2}^{\prime}\right)^{2}+4\left(\Delta_{2}-\Delta_{1}\right) \gamma_{1}^{\prime} \gamma_{2}^{\prime}\left(\gamma_{2}^{\prime} \gamma_{1}^{\prime \prime}+\gamma_{1}^{\prime} \gamma_{2}^{\prime \prime}\right)+o\left(\Delta_{12}\right)} \\
& +\frac{\Delta_{1}\left(-s^{2} \gamma_{2,-}^{\prime \prime \prime} \gamma_{1}^{\prime}-s^{2} \gamma_{1,-}^{\prime \prime \prime} \gamma_{2}^{\prime}+2 s s_{-}^{\prime \prime} \gamma_{2}^{\prime} \gamma_{1}^{\prime}\right)+2 \Delta_{2} s^{2} \gamma_{1}^{\prime \prime} \gamma_{2}^{\prime \prime}+o\left(\Delta_{12}\right)}{\left(2 \gamma_{1}^{\prime} \gamma_{2}^{\prime}\right)^{2}+4\left(\Delta_{2}-\Delta_{1}\right) \gamma_{1}^{\prime} \gamma_{2}^{\prime}\left(\gamma_{2}^{\prime} \gamma_{1}^{\prime \prime}+\gamma_{1}^{\prime} \gamma_{2}^{\prime \prime}\right)+o\left(\Delta_{12}\right)}
\end{aligned}
$$

We have

$$
-g_{2}\left(t_{1}, t_{2}\right)+g_{3}\left(t_{1}, t_{2}\right)=\frac{-g_{21}\left(t_{1}, t_{2}\right)+g_{31}\left(t_{1}, t_{2}\right)}{g_{22}\left(t_{1}, t_{2}\right)} .
$$

Then the approximation of the numerator of this quotient reads as

$$
\begin{aligned}
& -g_{21}\left(t_{1}, t_{2}\right)+g_{31}\left(t_{1}, t_{2}\right) \approx-s^{2} \operatorname{det}\left(\gamma^{\prime}, \gamma^{\prime \prime}\right)+\Delta_{1} s^{2} \operatorname{det}\left(\gamma^{\prime}, \gamma_{-}^{\prime \prime \prime}\right)-2 \Delta_{2} s s^{\prime} \operatorname{det}\left(\gamma^{\prime}, \gamma^{\prime \prime}\right) \\
& +s^{2} \gamma_{2}^{\prime \prime} \gamma_{1}^{\prime}+s^{2} \gamma_{2}^{\prime} \gamma_{1}^{\prime \prime}-2 s s^{\prime} \gamma_{2}^{\prime} \gamma_{1}^{\prime}-2 \Delta_{2}\left(s^{\prime}\right)^{2} \gamma_{2}^{\prime} \gamma_{1}^{\prime}+\Delta_{1}\left(-s^{2} \gamma_{2,-}^{\prime \prime \prime} \gamma_{1}^{\prime}\right. \\
& \left.-s^{2} \gamma_{1,-}^{\prime \prime \prime} \gamma_{2}^{\prime}+2 s s_{-}^{\prime \prime} \gamma_{2}^{\prime} \gamma_{1}^{\prime}\right)+2 \Delta_{2} s^{2} \gamma_{1}^{\prime \prime} \gamma_{2}^{\prime \prime} \\
& =-s^{2} \gamma_{2}^{\prime \prime} \gamma_{1}^{\prime}+s^{2} \gamma_{2}^{\prime} \gamma_{1}^{\prime \prime}+\Delta_{1} s^{2} \gamma_{2,-}^{\prime \prime \prime} \gamma_{1}^{\prime}-\Delta_{1} s^{2} \gamma_{1,-}^{\prime \prime \prime} \gamma_{2}^{\prime}-2 \Delta_{2} s s^{\prime} \gamma_{2}^{\prime \prime} \gamma_{1}^{\prime} \\
& +2 \Delta_{2} s s^{\prime} \gamma_{2}^{\prime} \gamma_{1}^{\prime \prime}+s^{2} \gamma_{2}^{\prime \prime} \gamma_{1}^{\prime}+s^{2} \gamma_{2}^{\prime} \gamma_{1}^{\prime \prime}-2 s s^{\prime} \gamma_{2}^{\prime} \gamma_{1}^{\prime}-2 \Delta_{2}\left(s^{\prime}\right)^{2} \gamma_{2}^{\prime} \gamma_{1}^{\prime} \\
& +\Delta_{1}\left(-s^{2} \gamma_{2,-}^{\prime \prime \prime} \gamma_{1}^{\prime}-s^{2} \gamma_{1,-}^{\prime \prime \prime} \gamma_{2}^{\prime}+2 s s_{-}^{\prime \prime} \gamma_{2}^{\prime} \gamma_{1}^{\prime}\right)+2 \Delta_{2} s^{2} \gamma_{1}^{\prime \prime} \gamma_{2}^{\prime \prime} \\
& =2 s^{2} \gamma_{2}^{\prime} \gamma_{1}^{\prime \prime}-2 \Delta_{1} s^{2} \gamma_{1,-}^{\prime \prime \prime} \gamma_{2}^{\prime}-2 \Delta_{2} s s^{\prime} \gamma_{2}^{\prime \prime} \gamma_{1}^{\prime}+2 \Delta_{2} s s^{\prime} \gamma_{2}^{\prime} \gamma_{1}^{\prime \prime}-2 s s^{\prime} \gamma_{2}^{\prime} \gamma_{1}^{\prime} \\
& -2 \Delta_{2}\left(s^{\prime}\right)^{2} \gamma_{2}^{\prime} \gamma_{1}^{\prime}+2 \Delta_{1} s s_{-}^{\prime \prime} \gamma_{2}^{\prime} \gamma_{1}^{\prime}+2 \Delta_{2} s^{2} \gamma_{1}^{\prime \prime} \gamma_{2}^{\prime \prime} \\
& =2 s^{2} \gamma_{2}^{\prime} \gamma_{1}^{\prime \prime}-2 s s^{\prime} \gamma_{2}^{\prime} \gamma_{1}^{\prime}-2 \Delta_{2} s s^{\prime} \operatorname{det}\left(\gamma^{\prime}, \gamma^{\prime \prime}\right)+2 \Delta_{2} s^{2} \gamma_{1}^{\prime \prime} \gamma_{2}^{\prime \prime} \\
& -2 \Delta_{2}\left(s^{\prime}\right)^{2} \gamma_{2}^{\prime} \gamma_{1}^{\prime}+2 \Delta_{1} s s_{-}^{\prime \prime} \gamma_{2}^{\prime} \gamma_{1}^{\prime}-2 \Delta_{1} s^{2} \gamma_{1,-}^{\prime \prime \prime} \gamma_{2}^{\prime} \\
& =2\left(\left(\gamma_{1}^{\prime}\right)^{2}+\left(\gamma_{2}^{\prime}\right)^{2}\right) \gamma_{2}^{\prime} \gamma_{1}^{\prime \prime} \\
& -2\left(\gamma_{1}^{\prime} \gamma_{1}^{\prime \prime}+\gamma_{2}^{\prime} \gamma_{2}^{\prime \prime}\right) \gamma_{2}^{\prime} \gamma_{1}^{\prime}-2 \Delta_{2}\left(\gamma_{1}^{\prime} \gamma_{1}^{\prime \prime}+\gamma_{2}^{\prime} \gamma_{2}^{\prime \prime}\right)\left(\gamma_{1}^{\prime} \gamma_{2}^{\prime \prime}-\gamma_{2}^{\prime} \gamma_{1}^{\prime \prime}\right) \\
& +2 \Delta_{2}\left(\left(\gamma_{1}^{\prime}\right)^{2}+\left(\gamma_{2}^{\prime}\right)^{2}\right) \gamma_{1}^{\prime \prime} \gamma_{2}^{\prime \prime}-2 \Delta_{2}\left(s^{\prime}\right)^{2} \gamma_{2}^{\prime} \gamma_{1}^{\prime}+2 \Delta_{1} s s_{-}^{\prime \prime} \gamma_{2}^{\prime} \gamma_{1}^{\prime} \\
& -2 \Delta_{1} s^{2} \gamma_{1,-}^{\prime \prime \prime} \gamma_{2}^{\prime} \\
& =2\left(\gamma_{1}^{\prime}\right)^{2} \gamma_{2}^{\prime} \gamma_{1}^{\prime \prime}+2\left(\gamma_{2}^{\prime}\right)^{3} \gamma_{1}^{\prime \prime}-2\left(\gamma_{1}^{\prime}\right)^{2} \gamma_{2}^{\prime} \gamma_{1}^{\prime \prime}-2\left(\gamma_{2}^{\prime}\right)^{2} \gamma_{2}^{\prime \prime} \gamma_{1}^{\prime} \\
& -2 \Delta_{2}\left(\left(\gamma_{1}^{\prime}\right)^{2} \gamma_{1}^{\prime \prime} \gamma_{2}^{\prime \prime}-\gamma_{1}^{\prime} \gamma_{2}^{\prime}\left(\gamma_{1}^{\prime \prime}\right)^{2}+\gamma_{2}^{\prime} \gamma_{1}^{\prime}\left(\gamma_{2}^{\prime \prime}\right)^{2}-\left(\gamma_{2}^{\prime}\right)^{2} \gamma_{2}^{\prime \prime} \gamma_{1}^{\prime \prime}\right) \\
& +2 \Delta_{2}\left(\left(\gamma_{1}^{\prime}\right)^{2} \gamma_{1}^{\prime \prime} \gamma_{2}^{\prime \prime}+\left(\gamma_{2}^{\prime}\right)^{2} \gamma_{1}^{\prime \prime} \gamma_{2}^{\prime \prime}\right)-2 \Delta_{2}\left(s^{\prime}\right)^{2} \gamma_{2}^{\prime} \gamma_{1}^{\prime}+2 \Delta_{1} s s_{-}^{\prime \prime} \gamma_{2}^{\prime} \gamma_{1}^{\prime} \\
& -2 \Delta_{1} s^{2} \gamma_{1,-}^{\prime \prime \prime} \gamma_{2}^{\prime} \\
& =-2\left(\gamma_{2}^{\prime}\right)^{2} \operatorname{det}\left(\gamma^{\prime}, \gamma^{\prime \prime}\right)+2 \Delta_{2} \gamma_{1}^{\prime} \gamma_{2}^{\prime}\left(\left(\gamma_{1}^{\prime \prime}\right)^{2}-\left(\gamma_{2}^{\prime \prime}\right)^{2}\right)+4 \Delta_{2}\left(\gamma_{2}^{\prime}\right)^{2} \gamma_{1}^{\prime \prime} \gamma_{2}^{\prime \prime} \\
& -2 \Delta_{2}\left(s^{\prime}\right)^{2} \gamma_{2}^{\prime} \gamma_{1}^{\prime}+2 \Delta_{1} s s_{-}^{\prime \prime} \gamma_{2}^{\prime} \gamma_{1}^{\prime}-2 \Delta_{1} s^{2} \gamma_{1,-}^{\prime \prime \prime} \gamma_{2}^{\prime} \\
& =-2\left(\gamma_{2}^{\prime}\right)^{2} \operatorname{det}\left(\gamma^{\prime}, \gamma^{\prime \prime}\right)+2 \Delta_{2} \gamma_{2}^{\prime} \gamma_{1}^{\prime \prime}\left\langle\gamma^{\prime}, \gamma^{\prime \prime}\right\rangle-2 \Delta_{2} \gamma_{2}^{\prime} \gamma_{2}^{\prime \prime} \operatorname{det}\left(\gamma^{\prime}, \gamma^{\prime \prime}\right) \\
& -2 \Delta_{2}\left(s^{\prime}\right)^{2} \gamma_{2}^{\prime} \gamma_{1}^{\prime}+2 \Delta_{1} s s_{-}^{\prime \prime} \gamma_{2}^{\prime} \gamma_{1}^{\prime}-2 \Delta_{1} s^{2} \gamma_{1,-}^{\prime \prime \prime} \gamma_{2}^{\prime} \text {. }
\end{aligned}
$$


After dividing by 2 the numerator and denominator of quotient 4.14) we can write the expansion for the partial derivative:

$$
\begin{aligned}
\frac{\partial G\left(t_{1}, t_{2}\right)}{\partial t_{1}}= & g_{1}\left(t_{1}, t_{2}\right)-g_{2}\left(t_{1}, t_{2}\right)+g_{3}\left(t_{1}, t_{2}\right) \\
= & \frac{\frac{1}{2} \operatorname{det}\left(\gamma^{\prime}, \gamma^{\prime \prime}\right)+\frac{\Delta_{2}^{3}}{6 \Delta^{2}} \operatorname{det}\left(\gamma^{\prime}, \gamma_{+}^{\prime \prime \prime}\right)+\frac{\Delta_{1}^{3}-3 \Delta \Delta_{1}^{2}}{6 \Delta^{2}} \operatorname{det}\left(\gamma^{\prime}, \gamma_{-}^{\prime \prime \prime}\right)+o\left(\Delta_{12}\right)}{\left(\gamma_{1}^{\prime}\right)^{2}+\left(\Delta_{2}-\Delta_{1}\right) \gamma_{1}^{\prime} \gamma_{1}^{\prime \prime}+o\left(\Delta_{12}\right)} \\
& +\frac{-\left(\gamma_{2}^{\prime}\right)^{2} \operatorname{det}\left(\gamma^{\prime}, \gamma^{\prime \prime}\right)+\Delta_{2} \gamma_{2}^{\prime} \gamma_{1}^{\prime \prime}\left\langle\gamma^{\prime}, \gamma^{\prime \prime}\right\rangle-\Delta_{1} \gamma_{2}^{\prime} \gamma_{1,-}^{\prime \prime \prime} s^{2}}{2\left(\gamma_{1}^{\prime} \gamma_{2}^{\prime}\right)^{2}+2\left(\Delta_{2}-\Delta_{1}\right) \gamma_{1}^{\prime} \gamma_{2}^{\prime}\left(\gamma_{2}^{\prime} \gamma_{1}^{\prime \prime}+\gamma_{1}^{\prime} \gamma_{2}^{\prime \prime}\right)+o\left(\Delta_{12}\right)} \\
& +\frac{-\Delta_{2} \gamma_{2}^{\prime} \gamma_{2}^{\prime \prime} \operatorname{det}\left(\gamma^{\prime}, \gamma^{\prime \prime}\right)-\Delta_{2} \gamma_{1}^{\prime} \gamma_{2}^{\prime}()^{\prime}+\Delta_{1} \gamma_{1}^{\prime} \gamma_{2}^{\prime} s s_{-}^{\prime \prime}+o\left(\Delta_{12}\right)}{2\left(\gamma_{1}^{\prime} \gamma_{2}^{\prime}\right)^{2}+2\left(\Delta_{2}-\Delta_{1}\right) \gamma_{1}^{\prime} \gamma_{2}^{\prime}\left(\gamma_{2}^{\prime} \gamma_{1}^{\prime \prime}+\gamma_{1}^{\prime} \gamma_{2}^{\prime \prime}\right)+o\left(\Delta_{12}\right)} .
\end{aligned}
$$

The approximations of the quotients in (4.15) are consistent by the order. We multiply the corresponding 1-jets and we find an approximation for the numerator and denominator of the resulting quotient in 4.15 :

$$
\begin{aligned}
& \left(\frac{1}{2} \operatorname{det}\left(\gamma^{\prime}, \gamma^{\prime \prime}\right)+\frac{\Delta_{2}^{3}}{6 \Delta^{2}} \operatorname{det}\left(\gamma^{\prime}, \gamma_{+}^{\prime \prime \prime}\right)+\frac{\Delta_{1}^{3}-3 \Delta_{1}^{2}}{6 \Delta^{2}} \operatorname{det}\left(\gamma^{\prime}, \gamma_{-}^{\prime \prime \prime}\right)\right) \otimes \\
& \otimes\left(2\left(\gamma_{1}^{\prime} \gamma_{2}^{\prime}\right)^{2}+2\left(\Delta_{2}-\Delta_{1}\right) \gamma_{1}^{\prime} \gamma_{2}^{\prime}\left(\gamma_{2}^{\prime} \gamma_{1}^{\prime \prime}+\gamma_{1}^{\prime} \gamma_{2}^{\prime \prime}\right)\right) \\
& +\left(\left(\gamma_{1}^{\prime}\right)^{2}+\left(\Delta_{2}^{-} \Delta_{1}\right) \gamma_{1}^{\prime} \gamma_{1}^{\prime \prime}\right) \otimes\left(-\left(\gamma_{2}^{\prime}\right)^{2} \operatorname{det}\left(\gamma^{\prime}, \gamma^{\prime \prime}\right)+\Delta_{2} \gamma_{2}^{\prime} \gamma_{1}^{\prime \prime}\left\langle\gamma^{\prime}, \gamma^{\prime \prime}\right\rangle-\Delta_{1} \gamma_{2}^{\prime} \gamma_{1,-}^{\prime \prime \prime} s^{2}\right. \\
& \left.-\Delta_{2} \gamma_{2}^{\prime} \gamma_{2}^{\prime \prime} \operatorname{det}\left(\gamma^{\prime}, \gamma^{\prime \prime}\right)-\Delta_{2} \gamma_{1}^{\prime} \gamma_{2}^{\prime}\left(s^{\prime}\right)^{2}+\Delta_{1} \gamma_{1}^{\prime} \gamma_{2}^{\prime} s s_{-}^{\prime \prime}\right) \\
& =\left(\gamma_{1}^{\prime} \gamma_{2}^{\prime}\right)^{2} \operatorname{det}\left(\gamma^{\prime}, \gamma^{\prime \prime}\right)+\left(\Delta_{2}-\Delta_{1}\right) \gamma_{1}^{\prime} \gamma_{2}^{\prime}\left(\gamma_{2}^{\prime} \gamma_{1}^{\prime \prime}+\gamma_{1}^{\prime} \gamma_{2}^{\prime \prime}\right) \operatorname{det}\left(\gamma^{\prime}, \gamma^{\prime \prime}\right) \\
& +\frac{\Delta_{2}^{3}}{3 \Delta^{2}}\left(\gamma_{1}^{\prime} \gamma_{2}^{\prime}\right)^{2} \operatorname{det}\left(\gamma^{\prime}, \gamma_{+}^{\prime \prime \prime}\right)+\frac{\Delta_{1}^{3}-3 \Delta \Delta_{1}^{2}}{3 \Delta^{2}}\left(\gamma_{1}^{\prime} \gamma_{2}^{\prime}\right)^{2} \operatorname{det}\left(\gamma^{\prime}, \gamma_{-}^{\prime \prime \prime}\right) \\
& -\left(\gamma_{1}^{\prime} \gamma_{2}^{\prime}\right)^{2} \operatorname{det}\left(\gamma^{\prime}, \gamma^{\prime \prime}\right)+\Delta_{2}\left(\gamma_{1}^{\prime}\right)^{2} \gamma_{2}^{\prime} \gamma_{1}^{\prime \prime}\left\langle\gamma^{\prime}, \gamma^{\prime \prime}\right\rangle-\Delta_{1}\left(\gamma_{1}^{\prime}\right)^{2} \gamma_{2}^{\prime} \gamma_{1,-}^{\prime \prime \prime} s^{2} \\
& -\Delta_{2}\left(\gamma_{1}^{\prime}\right)^{2} \gamma_{2}^{\prime} \gamma_{2}^{\prime \prime} \operatorname{det}\left(\gamma^{\prime}, \gamma^{\prime \prime}\right)-\Delta_{2}\left(\gamma_{1}^{\prime}\right)^{3} \gamma_{2}^{\prime}\left(s^{\prime}\right)^{2}+\Delta_{1}\left(\gamma_{1}^{\prime}\right)^{3} \gamma_{2}^{\prime} s s_{-}^{\prime \prime} \\
& -\left(\Delta_{2}^{-} \Delta_{1}\right)\left(\gamma_{2}^{\prime}\right)^{2} \gamma_{1}^{\prime} \gamma_{1}^{\prime \prime} \operatorname{det}\left(\gamma^{\prime}, \gamma^{\prime \prime}\right)=\gamma_{1}^{\prime} \gamma_{2}^{\prime}\left(\left(\Delta_{2}-\Delta_{1}\right)\left(\gamma_{2}^{\prime} \gamma_{1}^{\prime \prime}+\gamma_{1}^{\prime} \gamma_{2}^{\prime \prime}\right) \operatorname{det}\left(\gamma^{\prime}, \gamma^{\prime \prime}\right)\right. \\
& +\frac{\Delta_{2}^{3}}{3 \Delta^{2}}\left(\gamma_{1}^{\prime} \gamma_{2}^{\prime}\right) \operatorname{det}\left(\gamma^{\prime}, \gamma_{+}^{\prime \prime \prime}\right)+\frac{\Delta_{1}^{3}-3 \Delta \Delta_{1}^{2}}{3 \Delta^{2}}\left(\gamma_{1}^{\prime} \gamma_{2}^{\prime}\right) \operatorname{det}\left(\gamma^{\prime}, \gamma_{-}^{\prime \prime \prime}\right)+\Delta_{2} \gamma_{1}^{\prime} \gamma_{1}^{\prime \prime}\left\langle\gamma^{\prime}, \gamma^{\prime \prime}\right\rangle \\
& -\Delta_{1} \gamma_{1}^{\prime} \gamma_{1,-}^{\prime \prime \prime} s^{2}-\Delta_{2} \gamma_{1}^{\prime} \gamma_{2}^{\prime \prime} \operatorname{det}\left(\gamma^{\prime}, \gamma^{\prime \prime}\right)-\Delta_{2}\left(\gamma_{1}^{\prime}\right)^{2}\left(s^{\prime}\right)^{2}+\Delta_{1}\left(\gamma_{1}^{\prime}\right)^{2} s s_{-}^{\prime \prime} \\
& \left.-\left(\Delta_{2}^{-} \Delta_{1}\right) \gamma_{2}^{\prime} \gamma_{1}^{\prime \prime} \operatorname{det}\left(\gamma^{\prime}, \gamma^{\prime \prime}\right)\right)=\gamma_{1}^{\prime} \gamma_{2}^{\prime}\left(-\Delta_{1} \gamma_{1}^{\prime} \gamma_{2}^{\prime \prime} \operatorname{det}\left(\gamma^{\prime}, \gamma^{\prime \prime}\right)\right. \\
& +\frac{\Delta_{2}^{3}}{3 \Delta^{2}}\left(\gamma_{1}^{\prime} \gamma_{2}^{\prime}\right) \operatorname{det}\left(\gamma^{\prime}, \gamma_{+}^{\prime \prime \prime}\right)+\frac{\Delta_{1}^{3}-3 \Delta_{1}^{2}}{3 \Delta^{2}}\left(\gamma_{1}^{\prime} \gamma_{2}^{\prime}\right) \operatorname{det}\left(\gamma^{\prime}, \gamma_{-}^{\prime \prime \prime}\right) \\
& \left.+\Delta_{2} \gamma_{1}^{\prime} \gamma_{1}^{\prime \prime}\left\langle\gamma^{\prime}, \gamma^{\prime \prime}\right\rangle-\Delta_{1} \gamma_{1}^{\prime} \gamma_{1,-}^{\prime \prime \prime} s^{2}-\Delta_{2}\left(\gamma_{1}^{\prime}\right)^{2}\left(s^{\prime}\right)^{2}+\Delta_{1}\left(\gamma_{1}^{\prime}\right)^{2} s s_{-}^{\prime \prime}\right) \\
& =\left(\gamma_{1}^{\prime}\right)^{2} \gamma_{2}^{\prime}\left(-\Delta_{1} \gamma_{2}^{\prime \prime} \operatorname{det}\left(\gamma^{\prime}, \gamma^{\prime \prime}\right)+\frac{\Delta_{2}^{3}}{3 \Delta^{2}} \gamma_{2}^{\prime} \operatorname{det}\left(\gamma^{\prime}, \gamma_{+}^{\prime \prime \prime}\right)+\frac{\Delta_{1}^{3}-3 \Delta \Delta_{1}^{2}}{3 \Delta^{2}} \gamma_{2}^{\prime} \operatorname{det}\left(\gamma^{\prime}, \gamma_{-}^{\prime \prime \prime}\right)\right. \\
& \left.+\Delta_{1} \gamma_{1}^{\prime} s s_{-}^{\prime \prime}-\Delta_{1} \gamma_{1,-}^{\prime \prime \prime} s^{2}+\Delta_{2} \gamma_{1}^{\prime \prime}\left\langle\gamma^{\prime}, \gamma^{\prime \prime}\right\rangle-\Delta_{2} \gamma_{1}^{\prime}\left(s^{\prime}\right)^{2}\right) \\
& =\left(\gamma_{1}^{\prime}\right)^{2} \gamma_{2}^{\prime}\left(-\Delta_{1} \gamma_{2}^{\prime \prime} \operatorname{det}\left(\gamma^{\prime}, \gamma^{\prime \prime}\right)+\frac{\Delta_{2}^{3}}{3 \Delta^{2}} \gamma_{2}^{\prime} \operatorname{det}\left(\gamma^{\prime}, \gamma_{+}^{\prime \prime \prime}\right)+\frac{\Delta_{1}^{3}-3 \Delta \Delta_{1}^{2}}{3 \Delta^{2}} \gamma_{2}^{\prime} \operatorname{det}\left(\gamma^{\prime}, \gamma_{-}^{\prime \prime \prime}\right)\right. \\
& \left.+\Delta_{1} \gamma_{1}^{\prime} \frac{\left(\operatorname{det}\left(\gamma^{\prime}, \gamma^{\prime \prime}\right)\right)^{2}}{s^{2}}+\Delta_{1} \gamma_{2}^{\prime} \operatorname{det}\left(\gamma^{\prime}, \gamma_{-}^{\prime \prime \prime}\right)-\Delta_{2} \frac{\gamma_{2}^{\prime}\left\langle\gamma^{\prime}, \gamma^{\prime \prime}\right\rangle \operatorname{det}\left(\gamma^{\prime}, \gamma^{\prime \prime}\right)}{s^{2}}\right) \text {. }
\end{aligned}
$$


Here we have employed the following transformations:

$$
\begin{aligned}
\Delta_{2} \gamma_{1}^{\prime \prime}\left\langle\gamma^{\prime}, \gamma^{\prime \prime}\right\rangle-\Delta_{2} \gamma_{1}^{\prime}\left(s^{\prime}\right)^{2} & =\Delta_{2}\left(\gamma_{1}^{\prime \prime}\left\langle\gamma^{\prime}, \gamma^{\prime \prime}\right\rangle-\frac{\gamma_{1}^{\prime}\left\langle\gamma^{\prime}, \gamma^{\prime \prime}\right\rangle^{2}}{s^{2}}\right) \\
& =\Delta_{2} \frac{\gamma_{1}^{\prime \prime}\left(\gamma_{1}^{\prime} \gamma_{1}^{\prime \prime}+\gamma_{2}^{\prime} \gamma_{2}^{\prime \prime}\right)\left(\left(\gamma_{1}^{\prime}\right)^{2}+\left(\gamma_{2}^{\prime}\right)^{2}\right)-\gamma_{1}^{\prime}\left(\gamma_{1}^{\prime} \gamma_{1}^{\prime \prime}+\gamma_{2}^{\prime} \gamma_{2}^{\prime \prime}\right)^{2}}{s^{2}} \\
& =\Delta_{2} \frac{\left(\gamma_{1}^{\prime} \gamma_{1}^{\prime \prime}+\gamma_{2}^{\prime} \gamma_{2}^{\prime \prime}\right)\left(\gamma_{1}^{\prime \prime}\left(\gamma_{1}^{\prime}\right)^{2}+\gamma_{1}^{\prime \prime}\left(\gamma_{2}^{\prime}\right)^{2}-\left(\gamma_{1}^{\prime}\right)^{2} \gamma_{1}^{\prime \prime}-\gamma_{1}^{\prime} \gamma_{2}^{\prime} \gamma_{2}^{\prime \prime}\right)}{s^{2}} \\
& =\Delta_{2} \frac{\left(\gamma_{1}^{\prime} \gamma_{1}^{\prime \prime}+\gamma_{2}^{\prime} \gamma_{2}^{\prime \prime}\right) \gamma_{2}^{\prime}\left(\gamma_{1}^{\prime \prime} \gamma_{2}^{\prime}-\gamma_{1}^{\prime} \gamma_{2}^{\prime \prime}\right)}{s^{2}} \\
& =-\Delta_{2} \frac{\gamma_{2}^{\prime}\left\langle\gamma^{\prime}, \gamma^{\prime \prime}\right\rangle \operatorname{det}\left(\gamma^{\prime}, \gamma^{\prime \prime}\right)}{s^{2}}
\end{aligned}
$$

and

$$
\begin{aligned}
\Delta_{1} \gamma_{1}^{\prime} s s_{-}^{\prime \prime}-\Delta_{1} \gamma_{1,-}^{\prime \prime \prime} s^{2} & =\Delta_{1}\left(\gamma_{1}^{\prime} s s_{-}^{\prime \prime}-\gamma_{1,-}^{\prime \prime \prime} s^{2}\right) \\
& =\Delta_{1}\left(\gamma_{1}^{\prime} s\left(\frac{\left\langle\gamma^{\prime}, \gamma_{-}^{\prime \prime \prime}\right\rangle s^{2}+\left(\operatorname{det}\left(\gamma^{\prime}, \gamma^{\prime \prime}\right)\right)^{2}}{s^{3}}\right)-\gamma_{1,-}^{\prime \prime \prime} s^{2}\right) \\
& =\Delta_{1}\left(\gamma_{1}^{\prime}\left\langle\gamma^{\prime}, \gamma_{-}^{\prime \prime \prime}\right\rangle+\gamma_{1}^{\prime} \frac{\left(\operatorname{det}\left(\gamma^{\prime}, \gamma^{\prime \prime}\right)\right)^{2}}{s^{2}}-\gamma_{1,-}^{\prime \prime \prime} s^{2}\right) \\
& =\Delta_{1}\left(\gamma_{1}^{\prime}\left(\gamma_{1}^{\prime} \gamma_{1,-}^{\prime \prime \prime}+\gamma_{2}^{\prime} \gamma_{2,}^{\prime \prime \prime}\right)+\gamma_{1}^{\prime} \frac{\left(\operatorname{det}\left(\gamma^{\prime}, \gamma^{\prime \prime}\right)\right)^{2}}{s^{2}}-\gamma_{1,-}^{\prime \prime \prime}\left(\left(\gamma_{1}^{\prime}\right)^{2}+\left(\gamma_{2}^{\prime}\right)^{2}\right)\right) \\
& =\Delta_{1}\left(\left(\gamma_{1}^{\prime}\right)^{2} \gamma_{1,-}^{\prime \prime \prime}+\gamma_{1}^{\prime} \gamma_{2}^{\prime} \gamma_{2,-}^{\prime \prime \prime}+\gamma_{1}^{\prime} \frac{\left(\operatorname{det}\left(\gamma^{\prime}, \gamma^{\prime \prime}\right)\right)^{2}}{s^{2}}-\gamma_{1,-}^{\prime \prime \prime}\left(\gamma_{1}^{\prime}\right)^{2}-\gamma_{1,-}^{\prime \prime \prime}\left(\gamma_{2}^{\prime}\right)^{2}\right) \\
& =\Delta_{1}\left(\gamma_{1}^{\prime} \frac{\left(\operatorname{det}\left(\gamma^{\prime}, \gamma^{\prime \prime}\right)\right)^{2}}{s^{2}}+\gamma_{2}^{\prime} \operatorname{det}\left(\gamma^{\prime}, \gamma_{-}^{\prime \prime \prime}\right)\right) .
\end{aligned}
$$

We write the expansion for the partial derivative along the diffeomorphism, then we take $\Delta_{1}$ out the brackets and employing conditions (4.1), we divide the numerator and denominator by $\left(\gamma_{1}^{\prime}\right)^{2} \gamma_{2}^{\prime} \neq 0$ :

$$
\begin{aligned}
\frac{\partial G\left(t_{1}, t_{2}\left(t_{1}\right)\right)}{\partial t_{1}}= & \left(\gamma_{1}^{\prime}\right)^{2} \gamma_{2}^{\prime}\left(-\Delta_{1} \gamma_{2}^{\prime \prime} \operatorname{det}\left(\gamma^{\prime}, \gamma^{\prime \prime}\right)+\frac{\Delta_{2}^{3}}{3 \Delta^{2}} \gamma_{2}^{\prime} \operatorname{det}\left(\gamma^{\prime}, \gamma_{+}^{\prime \prime \prime}\right)\right. \\
& +\frac{\Delta_{1}^{3}-3 \Delta \Delta_{1}^{2}}{3 \Delta^{2}} \gamma_{2}^{\prime} \operatorname{det}\left(\gamma^{\prime}, \gamma_{-}^{\prime \prime \prime}\right)+\Delta_{1} \gamma_{1}^{\prime} \frac{\left(\operatorname{det}\left(\gamma^{\prime}, \gamma^{\prime \prime}\right)\right)^{2}}{s^{2}}+\Delta_{1} \gamma_{2}^{\prime} \operatorname{det}\left(\gamma^{\prime}, \gamma_{-}^{\prime \prime \prime}\right) \\
& \left.\left.-\Delta_{2} \frac{\gamma_{2}^{\prime}\left\langle\gamma^{\prime}, \gamma^{\prime \prime}\right\rangle \operatorname{det}\left(\gamma^{\prime}, \gamma^{\prime \prime}\right)}{s^{2}}\right)+o\left(\Delta_{1}\right)\right) \\
& \cdot\left(2\left(\gamma_{1}^{\prime}\right)^{4}\left(\gamma_{2}^{\prime}\right)^{2}+2\left(\Delta_{2}-\Delta_{1}\right)\left(\gamma_{1}^{\prime}\right)^{3} \gamma_{2}^{\prime}\left(2 \gamma_{2}^{\prime} \gamma_{1}^{\prime \prime}+\gamma_{1}^{\prime} \gamma_{2}^{\prime \prime}\right)\right)^{-1} \\
= & \Delta_{1}\left(-\gamma_{2}^{\prime \prime} \operatorname{det}\left(\gamma^{\prime}, \gamma^{\prime \prime}\right)+\frac{\Delta_{2}^{3}}{3 \Delta_{1} \Delta^{2}} \gamma_{2}^{\prime} \operatorname{det}\left(\gamma^{\prime}, \gamma_{+}^{\prime \prime \prime}\right)\right. \\
& +\frac{\Delta_{1}^{3}-3 \Delta \Delta_{1}^{2}}{3 \Delta_{1} \Delta^{2}} \gamma_{2}^{\prime} \operatorname{det}\left(\gamma^{\prime}, \gamma_{-}^{\prime \prime \prime}\right)+\gamma_{1}^{\prime} \frac{\left(\operatorname{det}\left(\gamma^{\prime}, \gamma^{\prime \prime}\right)\right)^{2}}{s^{2}}
\end{aligned}
$$




$$
\begin{aligned}
& \left.+\gamma_{2}^{\prime} \operatorname{det}\left(\gamma^{\prime}, \gamma_{-}^{\prime \prime \prime}\right)-\frac{\Delta_{2}}{\Delta_{1}} \frac{\gamma_{2}^{\prime}\left\langle\gamma^{\prime}, \gamma^{\prime \prime}\right\rangle \operatorname{det}\left(\gamma^{\prime}, \gamma^{\prime \prime}\right)}{s^{2}}+\varepsilon\left(\Delta_{1}\right)\right) \\
& \left(2\left(\gamma_{1}^{\prime}\right)^{2}\left(\gamma_{2}^{\prime}\right)+2\left(\Delta_{2}-\Delta_{1}\right)\left(\gamma_{1}^{\prime}\right)\left(2 \gamma_{2}^{\prime} \gamma_{1}^{\prime \prime}+\gamma_{1}^{\prime} \gamma_{2}^{\prime \prime}\right)\right)^{-1} .
\end{aligned}
$$

Let us find the limit of the quotient involved as a factor after the increment $\Delta_{1}$. While calculating this, we take into consideration expansions (4.7) and (4.8), which in particular imply the limiting identities:

$$
\lim _{\Delta_{1} \downarrow 0} \frac{\Delta_{2}}{\Delta_{1}}=-\lambda, \quad \lim _{\Delta_{1} \downarrow 0} \frac{\Delta}{\Delta_{1}}=1-\lambda, \quad \lim _{\Delta_{1} \downarrow 0} \frac{\Delta_{1}}{\Delta}=\frac{1}{1-\lambda}, \quad \lim _{\Delta_{1} \downarrow 0} \frac{\Delta_{2}}{\Delta}=\frac{-\lambda}{1-\lambda} .
$$

As a result we obtain:

$$
\begin{aligned}
& \lim _{\Delta_{1} \downarrow 0}\left(-\gamma_{2}^{\prime \prime} \operatorname{det}\left(\gamma^{\prime}, \gamma^{\prime \prime}\right)+\frac{\Delta_{2}^{3}}{3 \Delta_{1} \Delta^{2}} \gamma_{2}^{\prime} \operatorname{det}\left(\gamma^{\prime}, \gamma_{+}^{\prime \prime \prime}\right)+\frac{\Delta_{1}^{2}-3 \Delta \Delta_{1}}{3 \Delta^{2}} \gamma_{2}^{\prime} \operatorname{det}\left(\gamma^{\prime}, \gamma_{-}^{\prime \prime \prime}\right)\right) \\
& \left(2\left(\gamma_{1}^{\prime}\right)^{2} \gamma_{2}^{\prime}+2\left(\Delta_{2}-\Delta_{1}\right)\left(\gamma_{1}^{\prime}\right)\left(2 \gamma_{2}^{\prime} \gamma_{1}^{\prime \prime}+\gamma_{1}^{\prime} \gamma_{2}^{\prime \prime}\right)\right)^{-1} \\
& +\lim _{\Delta_{1} \downarrow 0}\left(\gamma_{1}^{\prime} \frac{\left(\operatorname{det}\left(\gamma^{\prime}, \gamma^{\prime \prime}\right)\right)^{2}}{s^{2}}+\gamma_{2}^{\prime} \operatorname{det}\left(\gamma^{\prime}, \gamma_{-}^{\prime \prime \prime}\right)-\frac{\Delta_{2}}{\Delta_{1}} \frac{\gamma_{2}^{\prime}\left\langle\gamma^{\prime}, \gamma^{\prime \prime}\right\rangle \operatorname{det}\left(\gamma^{\prime}, \gamma^{\prime \prime}\right)}{s^{2}}\right) \\
& \left(2\left(\gamma_{1}^{\prime}\right)^{2} \gamma_{2}^{\prime}+2\left(\Delta_{2}-\Delta_{1}\right)\left(\gamma_{1}^{\prime}\right)\left(2 \gamma_{2}^{\prime} \gamma_{1}^{\prime \prime}+\gamma_{1}^{\prime} \gamma_{2}^{\prime \prime}\right)\right)^{-1} \\
= & \left(-\gamma_{2}^{\prime \prime} \operatorname{det}\left(\gamma^{\prime}, \gamma^{\prime \prime}\right)+\frac{-\lambda^{3}}{3(1-\lambda)^{2}} \gamma_{2}^{\prime} \operatorname{det}\left(\gamma^{\prime}, \gamma_{+}^{\prime \prime \prime}\right)+\frac{3 \lambda-2}{3(1-\lambda)^{2}} \gamma_{2}^{\prime} \operatorname{det}\left(\gamma^{\prime}, \gamma_{-}^{\prime \prime \prime}\right)\right. \\
& \left.+\gamma_{1}^{\prime} \frac{\left(\operatorname{det}\left(\gamma^{\prime}, \gamma^{\prime \prime}\right)\right)^{2}}{s^{2}}+\gamma_{2}^{\prime} \operatorname{det}\left(\gamma^{\prime}, \gamma_{-}^{\prime \prime \prime}\right)+\lambda \frac{\gamma_{2}^{\prime}\left\langle\gamma^{\prime}, \gamma^{\prime \prime}\right\rangle \operatorname{det}\left(\gamma^{\prime}, \gamma^{\prime \prime}\right)}{s^{2}}\right)\left(2\left(\gamma_{1}^{\prime}\right)^{2} \gamma_{2}^{\prime}\right)^{-1} \\
= & \left(-\gamma_{2}^{\prime \prime} \operatorname{det}\left(\gamma^{\prime}, \gamma^{\prime \prime}\right)+\frac{-\lambda^{3}}{3(1-\lambda)^{2}} \gamma_{2}^{\prime} \operatorname{det}\left(\gamma^{\prime}, \gamma_{+}^{\prime \prime \prime}\right)+\frac{3 \lambda^{2}-3 \lambda+1}{3(1-\lambda)^{2}} \gamma_{2}^{\prime} \operatorname{det}\left(\gamma^{\prime}, \gamma_{-}^{\prime \prime \prime}\right)\right. \\
& \left.+\gamma_{1}^{\prime} \frac{\left(\operatorname{det}\left(\gamma^{\prime}, \gamma^{\prime \prime}\right)\right)^{2}}{s^{2}}+\lambda \frac{\gamma_{2}^{\prime}\left\langle\gamma^{\prime}, \gamma^{\prime \prime}\right\rangle \operatorname{det}\left(\gamma^{\prime}, \gamma^{\prime \prime}\right)}{s^{2}}\right)\left(2\left(\gamma_{1}^{\prime}\right)^{2} \gamma_{2}^{\prime}\right)^{-1} \cdot
\end{aligned}
$$

We have obtained a linear asymptotics of the function $\frac{\partial G\left(t_{1}, t_{2}\left(t_{1}\right)\right)}{\partial t_{1}}$ to left from $t_{0} \in \mathbb{R}$ :

$$
\frac{\partial G\left(t_{1}, t_{2}\left(t_{1}\right)\right)}{\partial t_{1}} \sim C_{1}\left(t_{0}-t_{1}\right), \quad C_{1}=\text { const }, \quad t_{1} \rightarrow t_{0}-0 .
$$

Here the scalar coefficient $C_{1}$ in the expansion is equal to limit (4.16). This implies:

$$
\lim _{t_{1} \rightarrow t_{0}-0} \frac{\partial G\left(t_{1}, t_{2}\left(t_{1}\right)\right)}{\partial t_{1}}=0 .
$$

This justifies the first identity in 4.3).

We proceed to $\frac{\partial G\left(t_{1}, t_{2}\right)}{\partial t_{2}}$. Since the function $G=G\left(t_{1}, t_{2}\right)$ is symmetric, then its partial derivatives are obtained by interchanging the variables; here

$$
\frac{\partial G\left(t_{2}, t_{1}\right)}{\partial t_{2}}=\frac{\partial G\left(t_{1}, t_{2}\right)}{\partial t_{1}}
$$

This is why

$$
\begin{aligned}
\frac{\partial G\left(t_{1}, t_{2}\right)}{\partial t_{2}}= & \frac{\operatorname{det}\left(\gamma^{\prime}\left(t_{2}\right), \gamma\left(t_{1}\right)-\gamma\left(t_{2}\right)\right)}{\left(\gamma_{1}\left(t_{2}\right)-\gamma_{1}\left(t_{1}\right)\right)^{2}}-\frac{\left(s\left(t_{1}\right)\right)^{2} \operatorname{det}\left(\gamma^{\prime}\left(t_{2}\right), \gamma^{\prime \prime}\left(t_{2}\right)\right)}{\left(\gamma_{2}^{\prime}\left(t_{1}\right) \gamma_{1}^{\prime}\left(t_{2}\right)+\gamma_{2}^{\prime}\left(t_{2}\right) \gamma_{1}^{\prime}\left(t_{1}\right)\right)^{2}} \\
& +\frac{s\left(t_{1}\right)\left(s\left(t_{2}\right)\left(\gamma_{2}^{\prime \prime}\left(t_{2}\right) \gamma_{1}^{\prime}\left(t_{1}\right)+\gamma_{2}^{\prime}\left(t_{1}\right) \gamma_{1}^{\prime \prime}\left(t_{2}\right)\right)-s^{\prime}\left(t_{2}\right)\left(\gamma_{2}^{\prime}\left(t_{1}\right) \gamma_{1}^{\prime}\left(t_{2}\right)+\gamma_{2}^{\prime}\left(t_{2}\right) \gamma_{1}^{\prime}\left(t_{1}\right)\right)\right)}{\left(\gamma_{2}^{\prime}\left(t_{1}\right) \gamma_{1}^{\prime}\left(t_{2}\right)+\gamma_{2}^{\prime}\left(t_{2}\right) \gamma_{1}^{\prime}\left(t_{1}\right)\right)^{2}}
\end{aligned}
$$


We introduce the notations:

$$
\frac{\partial G\left(t_{1}, t_{2}\right)}{\partial t_{2}}=q_{1}\left(t_{1}, t_{2}\right)-q_{2}\left(t_{1}, t_{2}\right)+q_{3}\left(t_{1}, t_{2}\right),
$$

where

$$
q_{1}\left(t_{1}, t_{2}\right)=\frac{q_{11}\left(t_{1}, t_{2}\right)}{q_{12}\left(t_{1}, t_{2}\right)}, \quad q_{2}\left(t_{1}, t_{2}\right)=\frac{q_{21}\left(t_{1}, t_{2}\right)}{q_{22}\left(t_{1}, t_{2}\right)}, \quad g_{3}\left(t_{1}, t_{2}\right)=\frac{q_{31}\left(t_{1}, t_{2}\right)}{q_{32}\left(t_{1}, t_{2}\right)}
$$

are the corresponding quotients in the expansion. The symmetry of our mathematical model simplifies essentially the approximation of these quotients. By the symmetry, while finding the expansions for the components $\frac{\partial G\left(t_{1}, t_{2}\right)}{\partial t_{2}}$, we should use the corresponding components $\frac{\partial G\left(t_{1}, t_{2}\right)}{\partial t_{1}}$ replacing here the increment $\Delta_{2}$ by $-\Delta_{1}, \Delta_{1}$ by $-\Delta_{2}, \Delta$ by $-\Delta, \gamma_{i,-}^{\prime \prime \prime}$ by $\gamma_{i,+}^{\prime \prime \prime}, \gamma_{i,+}^{\prime \prime \prime}$ by $\gamma_{i,-}^{\prime \prime \prime}$, $i=1,2$.

We have:

$$
\begin{aligned}
\operatorname{det}\left(\gamma^{\prime}\left(t_{2}\right), \gamma\left(t_{1}\right)-\gamma\left(t_{2}\right)\right)= & \Delta^{2}\left(\frac{1}{2} \operatorname{det}\left(\gamma^{\prime}, \gamma^{\prime \prime}\right)-\frac{\Delta_{1}^{3}}{6 \Delta^{2}} \operatorname{det}\left(\gamma^{\prime}, \gamma_{-}^{\prime \prime \prime}\right)+\frac{3 \Delta \Delta_{2}^{2}-\Delta_{2}^{3}}{6 \Delta^{2}} \operatorname{det}\left(\gamma^{\prime}, \gamma_{+}^{\prime \prime \prime}\right)\right) \\
& +o\left(\Delta_{12}^{3}\right) .
\end{aligned}
$$

Then

$$
\begin{aligned}
q_{1}\left(t_{1}, t_{2}\right) & =\frac{\Delta^{2}\left(\frac{\operatorname{det}\left(\gamma^{\prime}, \gamma^{\prime \prime}\right)}{2}+\frac{\Delta_{2}^{3}}{6 \Delta^{2}} \operatorname{det}\left(\gamma^{\prime}, \gamma_{+}^{\prime \prime \prime}\right)+\frac{\Delta_{1}^{3}-3 \Delta \Delta_{1}^{2}}{6 \Delta^{2}} \operatorname{det}\left(\gamma^{\prime}, \gamma_{-}^{\prime \prime \prime}\right)\right)+o\left(\Delta_{12}^{3}\right)}{\Delta^{2}\left(\left(\gamma_{1}^{\prime}\right)^{2}+\left(\Delta_{2}-\Delta_{1}\right) \gamma_{1}^{\prime} \gamma_{1}^{\prime \prime}\right)+o\left(\Delta_{12}^{3}\right)} \\
& =\frac{\frac{\operatorname{det}\left(\gamma^{\prime}, \gamma^{\prime \prime}\right)}{2}+\frac{\Delta_{2}^{3}}{6 \Delta^{2}} \operatorname{det}\left(\gamma^{\prime}, \gamma_{+}^{\prime \prime \prime}\right)+\frac{\Delta_{1}^{3}-3 \Delta \Delta_{1}^{2}}{6 \Delta^{2}} \operatorname{det}\left(\gamma^{\prime}, \gamma_{-}^{\prime \prime \prime}\right)+o\left(\Delta_{12}\right)}{\left(\gamma_{1}^{\prime}\right)^{2}+\left(\Delta_{2}-\Delta_{1}\right) \gamma_{1}^{\prime} \gamma_{1}^{\prime \prime}+o\left(\Delta_{12}\right)} .
\end{aligned}
$$

The approximation of the numerator of the second quotient $q_{2}\left(t_{1}, t_{2}\right)$ reads as

$$
\begin{aligned}
g_{21}\left(t_{1}, t_{2}\right) & =\left(s\left(t_{1}\right)\right)^{2} \operatorname{det}\left(\gamma^{\prime}\left(t_{2}\right), \gamma^{\prime \prime}\left(t_{2}\right)\right) \\
& =s^{2} \operatorname{det}\left(\gamma^{\prime}, \gamma^{\prime \prime}\right)+\Delta_{2} s^{2} \operatorname{det}\left(\gamma^{\prime}, \gamma_{+}^{\prime \prime \prime}\right)-2 \Delta_{1} s s^{\prime} \operatorname{det}\left(\gamma^{\prime}, \gamma^{\prime \prime}\right)+o\left(\Delta_{12}\right) .
\end{aligned}
$$

The approximation of the numerator of third quotient $g_{3}\left(t_{1}, t_{2}\right)$ is as follows:

$$
\begin{aligned}
q_{31}\left(t_{1}, t_{2}\right)= & \gamma_{2}^{\prime \prime} \gamma_{1}^{\prime} s^{2}+\gamma_{2}^{\prime} \gamma_{1}^{\prime \prime} s^{2}-2 \gamma_{2}^{\prime} \gamma_{1}^{\prime} s s^{\prime}+2 \Delta_{1} \gamma_{2}^{\prime} \gamma_{1}^{\prime}\left(s^{\prime}\right)^{2}-2 \Delta_{1} \gamma_{1}^{\prime \prime} \gamma_{2}^{\prime \prime} s^{2} \\
& +\Delta_{2} \gamma_{1}^{\prime} \gamma_{2,+}^{\prime \prime \prime} s^{2}+\Delta_{2} \gamma_{2}^{\prime} \gamma_{1,+}^{\prime \prime \prime} s^{2}-2 \Delta_{2} \gamma_{2}^{\prime} \gamma_{1}^{\prime} s s_{+}^{\prime \prime}+o\left(\Delta_{12}\right) .
\end{aligned}
$$

The numerator of the quotient

$$
-q_{2}\left(t_{1}, t_{2}\right)+q_{3}\left(t_{1}, t_{2}\right)=\frac{-q_{21}\left(t_{1}, t_{2}\right)+q_{31}\left(t_{1}, t_{2}\right)}{q_{22}\left(t_{1}, t_{2}\right)}
$$

is of the form:

$$
\begin{aligned}
-q_{21}\left(t_{1}, t_{2}\right)+q_{31}\left(t_{1}, t_{2}\right)= & -2\left(\gamma_{2}^{\prime}\right)^{2} \operatorname{det}\left(\gamma^{\prime}, \gamma^{\prime \prime}\right)+2 \Delta_{1} \gamma_{2}^{\prime} \gamma_{2}^{\prime \prime} \operatorname{det}\left(\gamma^{\prime}, \gamma^{\prime \prime}\right)-2 \Delta_{1} \gamma_{2}^{\prime} \gamma_{1}^{\prime \prime}\left\langle\gamma^{\prime}, \gamma^{\prime \prime}\right\rangle \\
& +2 \Delta_{1} \gamma_{2}^{\prime} \gamma_{1}^{\prime}\left(s^{\prime}\right)^{2}+2 \Delta_{2} \gamma_{2}^{\prime} \gamma_{1,+}^{\prime \prime \prime} s^{2}-2 \Delta_{2} \gamma_{2}^{\prime} \gamma_{1}^{\prime} s s_{+}^{\prime \prime}+o\left(\Delta_{12}\right) .
\end{aligned}
$$

We divide by 2 the numerator and denominator of the quotient being the second term and we obtain:

$$
\begin{aligned}
\frac{\partial G\left(t_{1}, t_{2}\right)}{\partial t_{2}}= & q_{1}\left(t_{1}, t_{2}\right)-q_{2}\left(t_{1}, t_{2}\right)+q_{3}\left(t_{1}, t_{2}\right) \\
= & \frac{\frac{\operatorname{det}\left(\gamma^{\prime}, \gamma^{\prime \prime}\right)}{2}-\frac{\Delta_{1}^{3}}{6 \Delta^{2}} \operatorname{det}\left(\gamma^{\prime}, \gamma_{-}^{\prime \prime \prime}\right)+\frac{3 \Delta \Delta_{2}^{2}-\Delta_{2}^{3}}{6 \Delta^{2}} \operatorname{det}\left(\gamma^{\prime}, \gamma_{+}^{\prime \prime \prime}\right)+o\left(\Delta_{12}\right)}{\left(\gamma_{1}^{\prime}\right)^{2}+\left(\Delta_{2}^{-} \Delta_{1}\right) \gamma_{1}^{\prime} \gamma_{1}^{\prime \prime}+o\left(\Delta_{12}\right)} \\
& +\left(-\left(\gamma_{2}^{\prime}\right)^{2} \operatorname{det}\left(\gamma^{\prime}, \gamma^{\prime \prime}\right)+\Delta_{1} \gamma_{2}^{\prime} \gamma_{2}^{\prime \prime} \operatorname{det}\left(\gamma^{\prime}, \gamma^{\prime \prime}\right)-\Delta_{1} \gamma_{2}^{\prime} \gamma_{1}^{\prime \prime}\left\langle\gamma^{\prime}, \gamma^{\prime \prime}\right\rangle\right. \\
& \left.+\Delta_{1} \gamma_{2}^{\prime} \gamma_{1}^{\prime}\left(s^{\prime}\right)^{2}+\Delta_{2} \gamma_{2}^{\prime} \gamma_{1,+}^{\prime \prime \prime} s^{2}-\Delta_{2} \gamma_{2}^{\prime} \gamma_{1}^{\prime} s s_{+}^{\prime \prime}+o\left(\Delta_{12}\right)\right)
\end{aligned}
$$




$$
\left(2\left(\gamma_{1}^{\prime} \gamma_{2}^{\prime}\right)^{2}+2\left(\Delta_{2}-\Delta_{1}\right) \gamma_{1}^{\prime} \gamma_{2}^{\prime}\left(\gamma_{2}^{\prime} \gamma_{1}^{\prime \prime}+\gamma_{1}^{\prime} \gamma_{2}^{\prime \prime}\right)+o\left(\Delta_{12}\right)\right)^{-1}
$$

The approximation of the quotients in the expansions are consistent by their orders. We multiply the corresponding 1-jets and find the approximations of the numerator and denominator of the resulting quotient:

$$
\begin{gathered}
\left(\frac{1}{2} \operatorname{det}\left(\gamma^{\prime}, \gamma^{\prime \prime}\right)-\frac{\Delta_{1}^{3}}{6 \Delta^{2}} \operatorname{det}\left(\gamma^{\prime}, \gamma_{-}^{\prime \prime \prime}\right)+\frac{3 \Delta \Delta_{2}^{2}-\Delta_{2}^{3}}{6 \Delta^{2}} \operatorname{det}\left(\gamma^{\prime}, \gamma_{+}^{\prime \prime \prime}\right)\right) \\
\otimes\left(2\left(\gamma_{1}^{\prime} \gamma_{2}^{\prime}\right)^{2}+2\left(\Delta_{2}-\Delta_{1}\right) \gamma_{1}^{\prime} \gamma_{2}^{\prime}\left(\gamma_{2}^{\prime} \gamma_{1}^{\prime \prime}+\gamma_{1}^{\prime} \gamma_{2}^{\prime \prime}\right)\right)+\left(\left(\gamma_{1}^{\prime}\right)^{2}+\left(\Delta_{2}^{-} \Delta_{1}\right) \gamma_{1}^{\prime} \gamma_{1}^{\prime \prime}\right) \\
\otimes\left(-\left(\gamma_{2}^{\prime}\right)^{2} \operatorname{det}\left(\gamma^{\prime}, \gamma^{\prime \prime}\right)+\Delta_{1} \gamma_{2}^{\prime} \gamma_{2}^{\prime \prime} \operatorname{det}\left(\gamma^{\prime}, \gamma^{\prime \prime}\right)-\Delta_{1} \gamma_{2}^{\prime} \gamma_{1}^{\prime \prime}\left\langle\gamma^{\prime}, \gamma^{\prime \prime}\right\rangle+\Delta_{1} \gamma_{2}^{\prime} \gamma_{1}^{\prime}\left(s^{\prime}\right)^{2}\right. \\
\left.\quad+\Delta_{2} \gamma_{2}^{\prime} \gamma_{1,+}^{\prime \prime \prime} s^{2}-\Delta_{2} \gamma_{2}^{\prime} \gamma_{1}^{\prime} s s_{+}^{\prime \prime}\right) \\
=\left(\gamma_{1}^{\prime}\right)^{2} \gamma_{2}^{\prime}\left(\Delta_{2} \gamma_{2}^{\prime \prime} \operatorname{det}\left(\gamma^{\prime}, \gamma^{\prime \prime}\right)-\frac{\Delta_{1}^{3}}{3 \Delta^{2}} \gamma_{2}^{\prime} \operatorname{det}\left(\gamma^{\prime}, \gamma_{-}^{\prime \prime \prime}\right)-\frac{\Delta_{2}^{3}-3 \Delta \Delta_{2}^{2}}{3 \Delta^{2}} \gamma_{2}^{\prime} \operatorname{det}\left(\gamma^{\prime}, \gamma_{+}^{\prime \prime \prime}\right)\right. \\
\left.\quad-\Delta_{2} \gamma_{1}^{\prime} \frac{\left(\operatorname{det}\left(\gamma^{\prime}, \gamma^{\prime \prime}\right)\right)^{2}}{s^{2}}-\Delta_{2} \gamma_{2}^{\prime} \operatorname{det}\left(\gamma^{\prime}, \gamma_{+}^{\prime \prime \prime}\right)+\Delta_{1} \frac{\gamma_{2}^{\prime}\left\langle\gamma^{\prime}, \gamma^{\prime \prime}\right\rangle \operatorname{det}\left(\gamma^{\prime}, \gamma^{\prime \prime}\right)}{s^{2}}\right) \\
=\left(\gamma_{1}^{\prime}\right)^{2} \gamma_{2}^{\prime}\left(-\Delta_{1} \gamma_{2}^{\prime \prime} \operatorname{det}\left(\gamma^{\prime}, \gamma^{\prime \prime}\right)+\frac{\Delta_{2}^{3}}{3 \Delta^{2}} \gamma_{2}^{\prime} \operatorname{det}\left(\gamma^{\prime}, \gamma_{+}^{\prime \prime \prime}\right)+\frac{\Delta_{1}^{3}-3 \Delta \Delta_{1}^{2}}{3 \Delta^{2}} \gamma_{2}^{\prime} \operatorname{det}\left(\gamma^{\prime}, \gamma_{-}^{\prime \prime \prime}\right)\right. \\
\left.\quad+\Delta_{1} \gamma_{1}^{\prime} s s_{-}^{\prime \prime}-\Delta_{1} \gamma_{1,-}^{\prime \prime \prime} s^{2}+\Delta_{2} \gamma_{1}^{\prime \prime}\left\langle\gamma^{\prime}, \gamma^{\prime \prime}\right\rangle-\Delta_{2} \gamma_{1}^{\prime}\left(s^{\prime}\right)^{2}\right) \\
=\left(\gamma_{1}^{\prime}\right)^{2} \gamma_{2}^{\prime}\left(-\Delta_{1} \gamma_{2}^{\prime \prime} \operatorname{det}\left(\gamma^{\prime}, \gamma^{\prime \prime}\right)+\frac{\Delta_{2}^{3}}{3 \Delta^{2}} \gamma_{2}^{\prime} \operatorname{det}\left(\gamma^{\prime}, \gamma_{+}^{\prime \prime \prime}\right)+\frac{\Delta_{1}^{3}-3 \Delta \Delta_{1}^{2}}{3 \Delta^{2}} \gamma_{2}^{\prime} \operatorname{det}\left(\gamma^{\prime}, \gamma_{-}^{\prime \prime \prime}\right)\right. \\
\left.\quad+\Delta_{1} \gamma_{1}^{\prime} \frac{\left(\operatorname{det}\left(\gamma^{\prime}, \gamma^{\prime \prime}\right)\right)^{2}}{s^{2}}+\Delta_{1} \gamma_{2}^{\prime} \operatorname{det}\left(\gamma^{\prime}, \gamma_{-}^{\prime \prime \prime}\right)-\Delta_{2} \frac{\gamma_{2}^{\prime}\left\langle\gamma^{\prime}, \gamma^{\prime \prime}\right\rangle \operatorname{det}\left(\gamma^{\prime}, \gamma^{\prime \prime}\right)}{s^{2}}\right) .
\end{gathered}
$$

We cancel out by $\left(\gamma_{1}^{\prime}\right)^{2} \gamma_{2}^{\prime} \neq 0$ and take $\Delta_{1}$ out the brackets. Then the expansion of the partial derivative becomes:

$$
\begin{aligned}
\frac{\partial G\left(t_{1}, t_{2}\left(t_{1}\right)\right)}{\partial t_{2}}=\Delta_{1} & \left(\left(\frac{\Delta_{2}}{\Delta_{1}} \gamma_{2}^{\prime \prime} \operatorname{det}\left(\gamma^{\prime}, \gamma^{\prime \prime}\right)-\frac{\Delta_{1}^{2}}{3 \Delta^{2}} \gamma_{2}^{\prime} \operatorname{det}\left(\gamma^{\prime}, \gamma_{-}^{\prime \prime \prime}\right)-\frac{\Delta_{2}^{3}-3 \Delta_{2}^{2}}{3 \Delta_{1} \Delta^{2}} \gamma_{2}^{\prime} \operatorname{det}\left(\gamma^{\prime}, \gamma_{+}^{\prime \prime \prime}\right)\right.\right. \\
- & \left.\left.\frac{\Delta_{2}}{\Delta_{1}} \gamma_{1}^{\prime} \frac{\left(\operatorname{det}\left(\gamma^{\prime}, \gamma^{\prime \prime}\right)\right)^{2}}{s^{2}}-\frac{\Delta_{2}}{\Delta_{1}} \gamma_{2}^{\prime} \operatorname{det}\left(\gamma^{\prime}, \gamma_{+}^{\prime \prime \prime}\right)+\frac{\gamma_{2}^{\prime}\left\langle\gamma^{\prime}, \gamma^{\prime \prime}\right\rangle \operatorname{det}\left(\gamma^{\prime}, \gamma^{\prime \prime}\right)}{s^{2}}\right)+\varepsilon\left(\Delta_{1}\right)\right) \\
& \left(2\left(\gamma_{1}^{\prime}\right)^{2} \gamma_{2}^{\prime}+2\left(\Delta_{2}-\Delta_{1}\right) \gamma_{1}^{\prime}\left(2 \gamma_{2}^{\prime} \gamma_{1}^{\prime \prime}+\gamma_{1}^{\prime} \gamma_{2}^{\prime \prime}\right)+\varepsilon\left(\Delta_{1}\right)\right)^{-1} .
\end{aligned}
$$

We find the limit of the quotient using (4.7), 4.8):

$$
\begin{aligned}
& \lim _{\Delta_{1} \downarrow 0}\left(\left(\frac{\Delta_{2}}{\Delta_{1}} \gamma_{2}^{\prime \prime} \operatorname{det}\left(\gamma^{\prime}, \gamma^{\prime \prime}\right)-\frac{\Delta_{1}^{2}}{3 \Delta^{2}} \gamma_{2}^{\prime} \operatorname{det}\left(\gamma^{\prime}, \gamma_{-}^{\prime \prime \prime}\right)-\frac{\Delta_{2}^{3}-3 \Delta_{2}^{2}}{3 \Delta_{1} \Delta^{2}} \gamma_{2}^{\prime} \operatorname{det}\left(\gamma^{\prime}, \gamma_{+}^{\prime \prime \prime}\right)\right.\right. \\
- & \left.\left.\frac{\Delta_{2}}{\Delta_{1}} \gamma_{1}^{\prime} \frac{\left(\operatorname{det}\left(\gamma^{\prime}, \gamma^{\prime \prime}\right)\right)^{2}}{s^{2}}-\frac{\Delta_{2}}{\Delta_{1}} \gamma_{2}^{\prime} \operatorname{det}\left(\gamma^{\prime}, \gamma_{+}^{\prime \prime \prime}\right)+\frac{\gamma_{2}^{\prime}\left\langle\gamma^{\prime}, \gamma^{\prime \prime}\right\rangle \operatorname{det}\left(\gamma^{\prime}, \gamma^{\prime \prime}\right)}{s^{2}}\right)+\varepsilon\left(\Delta_{1}\right)\right) \\
\left(2\left(\gamma_{1}^{\prime}\right)^{2} \gamma_{2}^{\prime}+2\left(\Delta_{2}-\Delta_{1}\right) \gamma_{1}^{\prime}\left(2 \gamma_{2}^{\prime} \gamma_{1}^{\prime \prime}+\gamma_{1}^{\prime} \gamma_{2}^{\prime \prime}\right)+\varepsilon\left(\Delta_{1}\right)\right)^{-1} & \left(-\lambda \gamma_{2}^{\prime \prime} \operatorname{det}\left(\gamma^{\prime}, \gamma^{\prime \prime}\right)-\frac{1}{3(1-\lambda)^{2}} \gamma_{2}^{\prime} \operatorname{det}\left(\gamma^{\prime}, \gamma_{-}^{\prime \prime \prime}\right)+\frac{\lambda^{3}-3 \lambda^{2}+3 \lambda}{3(1-\lambda)^{2}} \gamma_{2}^{\prime} \operatorname{det}\left(\gamma^{\prime}, \gamma_{+}^{\prime \prime \prime}\right)\right. \\
= & \left.+\lambda \gamma_{1}^{\prime} \frac{\left(\operatorname{det}\left(\gamma^{\prime}, \gamma^{\prime \prime}\right)\right)^{2}}{s^{2}}+\frac{\gamma_{2}^{\prime}\left\langle\gamma^{\prime}, \gamma^{\prime \prime}\right\rangle \operatorname{det}\left(\gamma^{\prime}, \gamma^{\prime \prime}\right)}{s^{2}}\right)\left(2\left(\gamma_{1}^{\prime}\right)^{2} \gamma_{2}^{\prime}\right)^{-1} .
\end{aligned}
$$


Hence, we obtain the equivalence of the function $\frac{\partial G\left(t_{1}, t_{2}\left(t_{1}\right)\right)}{\partial t_{2}}$ and the linear function to the left from $t_{0} \in \mathbb{R}$ :

$$
\frac{\partial G\left(t_{1}, t_{2}\left(t_{1}\right)\right)}{\partial t_{1}} \sim C_{2}\left(t_{0}-t_{1}\right), \quad C_{2}=\text { const }, \quad t_{1} \rightarrow t_{0}-0 .
$$

The scalar coefficient $C_{2}$ in the expansion is equal to limit (4.17). This implies

$$
\lim _{t_{1} \rightarrow t_{0}-0} \frac{\partial G\left(t_{1}, t_{2}\left(t_{1}\right)\right)}{\partial t_{2}}=0 .
$$

This justifies the second identity in 4.3).

After the substitution of coefficients (4.16) and (4.17) into condition (4.6) and collecting like terms in the numerator and denominator, this condition becomes:

$$
\begin{aligned}
\lambda= & -\gamma_{2}^{\prime \prime} \operatorname{det}\left(\gamma^{\prime}, \gamma^{\prime \prime}\right)+\frac{-\lambda^{3}}{3(1-\lambda)^{2}} \gamma_{2}^{\prime} \operatorname{det}\left(\gamma^{\prime}, \gamma_{+}^{\prime \prime \prime}\right)+\frac{3 \lambda^{2}-3 \lambda+1}{3(1-\lambda)^{2}} \gamma_{2}^{\prime} \operatorname{det}\left(\gamma^{\prime}, \gamma_{-}^{\prime \prime \prime}\right) \\
& \left.+\gamma_{1}^{\prime} \frac{\left(\operatorname{det}\left(\gamma^{\prime}, \gamma^{\prime \prime}\right)\right)^{2}}{s^{2}}+\lambda \frac{\gamma_{2}^{\prime}\left\langle\gamma^{\prime}, \gamma^{\prime \prime}\right\rangle \operatorname{det}\left(\gamma^{\prime}, \gamma^{\prime \prime}\right)}{s^{2}}\right) \\
& \left(-\lambda \gamma_{2}^{\prime \prime} \operatorname{det}\left(\gamma^{\prime}, \gamma^{\prime \prime}\right)-\frac{1}{3(1-\lambda)^{2}} \gamma_{2}^{\prime} \operatorname{det}\left(\gamma^{\prime}, \gamma_{-}^{\prime \prime \prime}\right)+\frac{\lambda^{3}-3 \lambda^{2}+3 \lambda}{3(1-\lambda)^{2}} \gamma_{2}^{\prime} \operatorname{det}\left(\gamma^{\prime}, \gamma_{+}^{\prime \prime \prime}\right)\right. \\
& \left.+\lambda \gamma_{1}^{\prime} \frac{\left(\operatorname{det}\left(\gamma^{\prime}, \gamma^{\prime \prime}\right)\right)^{2}}{s^{2}}+\frac{\gamma_{2}^{\prime}\left\langle\gamma^{\prime}, \gamma^{\prime \prime}\right\rangle \operatorname{det}\left(\gamma^{\prime}, \gamma^{\prime \prime}\right)}{s^{2}}\right)^{-1} .
\end{aligned}
$$

Then

$$
\begin{aligned}
& \gamma_{2}^{\prime \prime} \operatorname{det}\left(\gamma^{\prime}, \gamma^{\prime \prime}\right)+\frac{\lambda^{3}}{3(1-\lambda)^{2}} \gamma_{2}^{\prime} \operatorname{det}\left(\gamma^{\prime}, \gamma_{+}^{\prime \prime \prime}\right)-\frac{3 \lambda^{2}-3 \lambda+1}{3(1-\lambda)^{2}} \gamma_{2}^{\prime} \operatorname{det}\left(\gamma^{\prime}, \gamma_{-}^{\prime \prime \prime}\right) \\
& -\gamma_{1}^{\prime} \frac{\left(\operatorname{det}\left(\gamma^{\prime}, \gamma^{\prime \prime}\right)\right)^{2}}{s^{2}}-\lambda \frac{\gamma_{2}^{\prime}\left\langle\gamma^{\prime}, \gamma^{\prime \prime}\right\rangle \operatorname{det}\left(\gamma^{\prime}, \gamma^{\prime \prime}\right)}{s^{2}} \\
= & -\lambda^{2} \gamma_{2}^{\prime \prime} \operatorname{det}\left(\gamma^{\prime}, \gamma^{\prime \prime}\right)-\frac{\lambda}{3(1-\lambda)^{2}} \gamma_{2}^{\prime} \operatorname{det}\left(\gamma^{\prime}, \gamma_{-}^{\prime \prime \prime}\right)+\lambda \frac{\lambda^{3}-3 \lambda^{2}+3 \lambda}{3(1-\lambda)^{2}} \gamma_{2}^{\prime} \operatorname{det}\left(\gamma^{\prime}, \gamma_{+}^{\prime \prime \prime}\right) \\
& +\lambda^{2} \gamma_{1}^{\prime} \frac{\left(\operatorname{det}\left(\gamma^{\prime}, \gamma^{\prime \prime}\right)\right)^{2}}{s^{2}}+\lambda \frac{\gamma_{2}^{\prime}\left\langle\gamma^{\prime}, \gamma^{\prime \prime}\right\rangle \operatorname{det}\left(\gamma^{\prime}, \gamma^{\prime \prime}\right)}{s^{2}}
\end{aligned}
$$

and

$$
\begin{aligned}
& \gamma_{2}^{\prime \prime} \operatorname{det}\left(\gamma^{\prime}, \gamma^{\prime \prime}\right)+\frac{\lambda^{3}}{3(1-\lambda)^{2}} \gamma_{2}^{\prime} \operatorname{det}\left(\gamma^{\prime}, \gamma_{+}^{\prime \prime \prime}\right)-\frac{3 \lambda^{2}-3 \lambda+1}{3(1-\lambda)^{2}} \gamma_{2}^{\prime} \operatorname{det}\left(\gamma^{\prime}, \gamma_{-}^{\prime \prime \prime}\right) \\
& -\gamma_{1}^{\prime} \frac{\left(\operatorname{det}\left(\gamma^{\prime}, \gamma^{\prime \prime}\right)\right)^{2}}{s^{2}}-\lambda \frac{\gamma_{2}^{\prime}\left\langle\gamma^{\prime}, \gamma^{\prime \prime}\right\rangle \operatorname{det}\left(\gamma^{\prime}, \gamma^{\prime \prime}\right)}{s^{2}}+\lambda^{2} \gamma_{2}^{\prime \prime} \operatorname{det}\left(\gamma^{\prime}, \gamma^{\prime \prime}\right) \\
& +\frac{\lambda}{3(1-\lambda)^{2}} \gamma_{2}^{\prime} \operatorname{det}\left(\gamma^{\prime}, \gamma_{-}^{\prime \prime \prime}\right)-\lambda \frac{\lambda^{3}-3 \lambda^{2}+3 \lambda}{3(1-\lambda)^{2}} \gamma_{2}^{\prime} \operatorname{det}\left(\gamma^{\prime}, \gamma_{+}^{\prime \prime \prime}\right) \\
& -\lambda^{2} \gamma_{1}^{\prime} \frac{\left(\operatorname{det}\left(\gamma^{\prime}, \gamma^{\prime \prime}\right)\right)^{2}}{s^{2}}-\lambda \frac{\gamma_{2}^{\prime}\left\langle\gamma^{\prime}, \gamma^{\prime \prime}\right\rangle \operatorname{det}\left(\gamma^{\prime}, \gamma^{\prime \prime}\right)}{s^{2}}=0 .
\end{aligned}
$$

We also have:

$$
\begin{aligned}
& \lambda^{2}\left(\frac{\lambda}{3(1-\lambda)^{2}}-\frac{\lambda^{2}-3 \lambda+3}{3(1-\lambda)^{2}}\right) \gamma_{2}^{\prime} \operatorname{det}\left(\gamma^{\prime}, \gamma_{+}^{\prime \prime \prime}\right) \\
& +\left(\frac{\lambda}{3(1-\lambda)^{2}}-\frac{3 \lambda^{2}-3 \lambda+1}{3(1-\lambda)^{2}}\right) \gamma_{2}^{\prime} \operatorname{det}\left(\gamma^{\prime}, \gamma_{-}^{\prime \prime \prime}\right)+\left(\lambda^{2}+1\right) \gamma_{2}^{\prime \prime} \operatorname{det}\left(\gamma^{\prime}, \gamma^{\prime \prime}\right)
\end{aligned}
$$




$$
-\left(\lambda^{2}+1\right) \gamma_{1}^{\prime} \frac{\left(\operatorname{det}\left(\gamma^{\prime}, \gamma^{\prime \prime}\right)\right)^{2}}{s^{2}}-2 \lambda \frac{\gamma_{2}^{\prime}\left\langle\gamma^{\prime}, \gamma^{\prime \prime}\right\rangle \operatorname{det}\left(\gamma^{\prime}, \gamma^{\prime \prime}\right)}{s^{2}}=0
$$

Since

$$
\begin{aligned}
& \frac{\lambda}{3(1-\lambda)^{2}}-\frac{\lambda^{2}-3 \lambda+3}{3(1-\lambda)^{2}}=-\frac{\lambda^{2}-4 \lambda+3}{3(1-\lambda)^{2}}=-\frac{(\lambda-1)(\lambda-3)}{3(1-\lambda)^{2}}=\frac{\lambda-3}{3(1-\lambda)}, \\
& \frac{\lambda}{3(1-\lambda)^{2}}-\frac{3 \lambda^{2}-3 \lambda+1}{3(1-\lambda)^{2}}=\frac{\lambda^{2}-\frac{4}{3} \lambda+\frac{1}{3}}{-(1-\lambda)^{2}}=\frac{\left(\lambda-\frac{1}{3}\right)(\lambda-1)}{-(1-\lambda)^{2}}=\frac{3 \lambda-1}{3(1-\lambda)},
\end{aligned}
$$

then

$$
\begin{aligned}
& \lambda^{2} \frac{\lambda-3}{3(1-\lambda)} \gamma_{2}^{\prime} \operatorname{det}\left(\gamma^{\prime}, \gamma_{+}^{\prime \prime \prime}\right)+\frac{3 \lambda-1}{3(1-\lambda)} \gamma_{2}^{\prime} \operatorname{det}\left(\gamma^{\prime}, \gamma_{-}^{\prime \prime \prime}\right) \\
& +\left(\lambda^{2}+1\right)\left(\gamma_{2}^{\prime \prime} \operatorname{det}\left(\gamma^{\prime}, \gamma^{\prime \prime}\right)-\gamma_{1}^{\prime} \frac{\left(\operatorname{det}\left(\gamma^{\prime}, \gamma^{\prime \prime}\right)\right)^{2}}{s^{2}}\right)-2 \lambda \frac{\gamma_{2}^{\prime}\left\langle\gamma^{\prime}, \gamma^{\prime \prime}\right\rangle \operatorname{det}\left(\gamma^{\prime}, \gamma^{\prime \prime}\right)}{s^{2}}=0
\end{aligned}
$$

We make algebraic transformations:

$$
\begin{aligned}
\gamma_{2}^{\prime \prime} \operatorname{det}\left(\gamma^{\prime}, \gamma^{\prime \prime}\right)-\gamma_{1}^{\prime} \frac{\left(\operatorname{det}\left(\gamma^{\prime}, \gamma^{\prime \prime}\right)\right)^{2}}{s^{2}} & =\operatorname{det}\left(\gamma^{\prime}, \gamma^{\prime \prime}\right)\left(\gamma_{2}^{\prime \prime}-\gamma_{1}^{\prime} \frac{\operatorname{det}\left(\gamma^{\prime}, \gamma^{\prime \prime}\right)}{s^{2}}\right) \\
& =\operatorname{det}\left(\gamma^{\prime}, \gamma^{\prime \prime}\right) \frac{\gamma_{2}^{\prime \prime}\left(\left(\gamma_{1}^{\prime}\right)^{2}+\left(\gamma_{2}^{\prime}\right)^{2}\right)-\gamma_{1}^{\prime}\left(\gamma_{1}^{\prime} \gamma_{2}^{\prime \prime}-\gamma_{2}^{\prime} \gamma_{1}^{\prime \prime}\right)}{s^{2}} \\
& =\operatorname{det}\left(\gamma^{\prime}, \gamma^{\prime \prime}\right) \frac{\gamma_{2}^{\prime \prime}\left(\gamma_{1}^{\prime}\right)^{2}+\gamma_{2}^{\prime \prime}\left(\gamma_{2}^{\prime}\right)^{2}-\gamma_{2}^{\prime \prime}\left(\gamma_{1}^{\prime}\right)^{2}+\gamma_{1}^{\prime} \gamma_{2}^{\prime} \gamma_{1}^{\prime \prime}}{s^{2}} \\
& =\operatorname{det}\left(\gamma^{\prime}, \gamma^{\prime \prime}\right) \frac{\gamma_{2}^{\prime \prime}\left(\gamma_{2}^{\prime}\right)^{2}+\gamma_{1}^{\prime} \gamma_{2}^{\prime} \gamma_{1}^{\prime \prime}}{s^{2}} \\
& =\frac{\gamma_{2}^{\prime} \operatorname{det}\left(\gamma^{\prime}, \gamma^{\prime \prime}\right)\left\langle\gamma^{\prime}, \gamma^{\prime \prime}\right\rangle}{s^{2}} .
\end{aligned}
$$

Then

$$
\begin{aligned}
& \lambda^{2} \frac{\lambda-3}{3(1-\lambda)} \gamma_{2}^{\prime} \operatorname{det}\left(\gamma^{\prime}, \gamma_{+}^{\prime \prime \prime}\right)+\frac{3 \lambda-1}{3(1-\lambda)} \gamma_{2}^{\prime} \operatorname{det}\left(\gamma^{\prime}, \gamma_{-}^{\prime \prime \prime}\right)+\left(\lambda^{2}+1\right) \frac{\gamma_{2}^{\prime} \operatorname{det}\left(\gamma^{\prime}, \gamma^{\prime \prime}\right)\left\langle\gamma^{\prime}, \gamma^{\prime \prime}\right\rangle}{s^{2}} \\
& -2 \lambda \frac{\gamma_{2}^{\prime}\left\langle\gamma^{\prime}, \gamma^{\prime \prime}\right\rangle \operatorname{det}\left(\gamma^{\prime}, \gamma^{\prime \prime}\right)}{s^{2}}=0 \\
& \lambda^{2} \frac{\lambda-3}{3(1-\lambda)} \gamma_{2}^{\prime} \operatorname{det}\left(\gamma^{\prime}, \gamma_{+}^{\prime \prime \prime}\right)+\frac{3 \lambda-1}{3(1-\lambda)} \gamma_{2}^{\prime} \operatorname{det}\left(\gamma^{\prime}, \gamma_{-}^{\prime \prime \prime}\right) \\
& +\frac{\gamma_{2}^{\prime} \operatorname{det}\left(\gamma^{\prime}, \gamma^{\prime \prime}\right)\left\langle\gamma^{\prime}, \gamma^{\prime \prime}\right\rangle}{s^{2}}\left(\lambda^{2}+1-2 \lambda\right)=0 \\
& \lambda^{2} \frac{\lambda-3}{3(1-\lambda)} \gamma_{2}^{\prime} \operatorname{det}\left(\gamma^{\prime}, \gamma_{+}^{\prime \prime \prime}\right)+\frac{3 \lambda-1}{3(1-\lambda)} \gamma_{2}^{\prime} \operatorname{det}\left(\gamma^{\prime}, \gamma_{-}^{\prime \prime \prime}\right) \\
& +\frac{\gamma_{2}^{\prime} \operatorname{det}\left(\gamma^{\prime}, \gamma^{\prime \prime}\right)\left\langle\gamma^{\prime}, \gamma^{\prime \prime}\right\rangle}{s^{2}}(\lambda-1)^{2}=0 \\
& \lambda^{2}(\lambda-3) \gamma_{2}^{\prime} \operatorname{det}\left(\gamma^{\prime}, \gamma_{+}^{\prime \prime \prime}\right)+(3 \lambda-1) \gamma_{2}^{\prime} \operatorname{det}\left(\gamma^{\prime}, \gamma_{-}^{\prime \prime \prime}\right)=\frac{3 \gamma_{2}^{\prime} \operatorname{det}\left(\gamma^{\prime}, \gamma^{\prime \prime}\right)\left\langle\gamma^{\prime}, \gamma^{\prime \prime}\right\rangle}{s^{2}}(\lambda-1)^{3} .
\end{aligned}
$$

We cancel out by $\gamma_{2}^{\prime} \neq 0$ :

$$
\lambda^{2}(\lambda-3) \operatorname{det}\left(\gamma^{\prime}, \gamma_{+}^{\prime \prime \prime}\right)+(3 \lambda-1) \operatorname{det}\left(\gamma^{\prime}, \gamma_{-}^{\prime \prime \prime}\right)=\frac{3 \operatorname{det}\left(\gamma^{\prime}, \gamma^{\prime \prime}\right)\left\langle\gamma^{\prime}, \gamma^{\prime \prime}\right\rangle}{s^{2}}(\lambda-1)^{3} .
$$

This proves identity 4.4 and completes the proof of the theorem. 


\section{Corollary From theOREM}

Corollary 5.1. Under the assumptions of Theorem 4.1, identity (4.4) is equivalent to the identity

$$
\left(\lambda^{3}-3 \lambda^{2}\right) k_{+}^{\prime}+(3 \lambda-1) k_{-}^{\prime}=0,
$$

where $k_{-}^{\prime}=k^{\prime}\left(t_{0}-0\right)$ is the one-sided left curvature, $k_{+}^{\prime}=k^{\prime}\left(t_{0}+0\right)$ is the one-sided right curvature of the curve $\Gamma$ at the point $\mathbf{x}^{(0)}=\left(\gamma_{1}\left(t_{0}\right), \gamma_{2}\left(t_{0}\right)\right)$.

Proof. In equation (4.4) we collect the terms with like powers of the left marker and then we regroup the terms:

$$
\begin{aligned}
& \lambda^{2}(\lambda-3) \operatorname{det}\left(\gamma^{\prime}, \gamma_{+}^{\prime \prime \prime}\right)+(3 \lambda-1) \operatorname{det}\left(\gamma^{\prime}, \gamma_{-}^{\prime \prime \prime}\right)-\frac{3 \operatorname{det}\left(\gamma^{\prime}, \gamma^{\prime \prime}\right)\left\langle\gamma^{\prime}, \gamma^{\prime \prime}\right\rangle}{s^{2}}(\lambda-1)^{3}=0, \\
& \begin{aligned}
\lambda^{2}(\lambda-3) & \left(\operatorname{det}\left(\gamma^{\prime}, \gamma_{+}^{\prime \prime \prime}\right)-\frac{3 \operatorname{det}\left(\gamma^{\prime}, \gamma^{\prime \prime}\right)\left\langle\gamma^{\prime}, \gamma^{\prime \prime}\right\rangle}{s^{2}}\right) \\
& +(3 \lambda-1)\left(\operatorname{det}\left(\gamma^{\prime}, \gamma_{-}^{\prime \prime \prime}\right)-\frac{3 \operatorname{det}\left(\gamma^{\prime}, \gamma^{\prime \prime}\right)\left\langle\gamma^{\prime}, \gamma^{\prime \prime}\right\rangle}{s^{2}}\right)=0, \\
\lambda^{2}(\lambda-3) & \left(\operatorname{det}\left(\gamma^{\prime}, \gamma_{+}^{\prime \prime \prime}\right) s^{2}-3 \operatorname{det}\left(\gamma^{\prime}, \gamma^{\prime \prime}\right)\left\langle\gamma^{\prime}, \gamma^{\prime \prime}\right\rangle\right) \\
& +(3 \lambda-1)\left(\operatorname{det}\left(\gamma^{\prime}, \gamma_{-}^{\prime \prime \prime}\right) s^{2}-3 \operatorname{det}\left(\gamma^{\prime}, \gamma^{\prime \prime}\right)\left\langle\gamma^{\prime}, \gamma^{\prime \prime}\right\rangle\right)=0 .
\end{aligned}
\end{aligned}
$$

It remains to note the square brackets contain the numerators of the corresponding one-sided derivatives of the curvature

$$
k_{ \pm}^{\prime}=\frac{\operatorname{det}\left(\gamma^{\prime}, \gamma_{ \pm}^{\prime \prime \prime}\right) s^{2}-3 \operatorname{det}\left(\gamma^{\prime}, \gamma^{\prime \prime}\right)\left\langle\gamma^{\prime}, \gamma^{\prime \prime}\right\rangle}{s^{5}}
$$

We divide the left part of 5.2 by $s^{5} \neq 0$ and we obtain (5.1).

We introduce algebraic equations, the real roots of which are mutual reciprocal.

Definition 5.1. For an arbitrary triple of real numbers $a, b, c$, two algebraic third order equations with respect to $z$

$$
\begin{aligned}
& a z^{2}(z-3)+b(3 z-1)+c(z-1)^{3}=0, \\
& b z^{2}(z-3)+a(3 z-1)+c(z-1)^{3}=0,
\end{aligned}
$$

are called adjoint equations.

Lemma 5.1. If $\lambda \neq 0$ is a real root of the equation

$$
a z^{2}(z-3)+b(3 z-1)+c(z-1)^{3}=0,
$$

then the reciprocal $\mu=\lambda^{-1}$ is a root of the adjoint equation

$$
b z^{2}(z-3)+a(3 z-1)+c(z-1)^{3}=0 .
$$

Proof. By the assumption, $\lambda=\mu^{-1}$ is the root of equation (5.3):

$$
a \mu^{-2}\left(\mu^{-1}-3\right)+b\left(3 \mu^{-1}-1\right)+c\left(\mu^{-1}-1\right)^{3}=0 .
$$

We transform this identity:

$$
\begin{aligned}
& a \frac{1-3 \mu}{\mu^{3}}+b \frac{3-\mu}{\mu}+c\left(\frac{1-\mu}{\mu}\right)^{3}=0, \\
& a \frac{1-3 \mu}{\mu^{3}}+b \frac{3 \mu^{2}-\mu^{3}}{\mu^{3}}+c \frac{(1-\mu)^{3}}{\mu^{3}}=0, \\
& \frac{-a(3 \mu-1)-b\left(\mu^{3}-3 \mu^{2}\right)-c(\mu-1)^{3}}{\mu^{3}}=0 .
\end{aligned}
$$


After dividing by $-\mu^{-3} \neq 0$ we get:

$$
b\left(\mu^{3}-3 \mu^{2}\right)+a(3 \mu-1)+c(\mu-1)^{3}=0 .
$$

Thus, $\mu=\lambda^{-1}$ solves equation (5.4).

Corollary 5.2. Under the assumptions of Theorem 4.1, the right marker $\mu=t_{1}^{\prime}\left(t_{0}+0\right)$ of the pseudo-vertex satisfies the identity

$$
\mu^{2}(\mu-3) \operatorname{det}\left(\gamma^{\prime}, \gamma_{-}^{\prime \prime \prime}\right)+(3 \mu-1) \operatorname{det}\left(\gamma^{\prime}, \gamma_{+}^{\prime \prime \prime}\right)=\frac{3 \operatorname{det}\left(\gamma^{\prime}, \gamma^{\prime \prime}\right)\left\langle\gamma^{\prime}, \gamma^{\prime \prime}\right\rangle}{s^{2}}(\mu-1)^{3} .
$$

Proof. By Theorem 4.1, the left marker $\lambda=t_{2}^{\prime}\left(t_{0}-0\right)$ satisfies the identity

$$
\lambda^{2}(\lambda-3) \operatorname{det}\left(\gamma^{\prime}, \gamma_{+}^{\prime \prime \prime}\right)+(3 \lambda-1) \operatorname{det}\left(\gamma^{\prime}, \gamma_{-}^{\prime \prime \prime}\right)=\frac{3 \operatorname{det}\left(\gamma^{\prime}, \gamma^{\prime \prime}\right)\left\langle\gamma^{\prime}, \gamma^{\prime \prime}\right\rangle}{s^{2}}(\lambda-1)^{3} .
$$

In other words, it is a root of equation $\sqrt{5.3}$ with the coefficients

$$
a=\operatorname{det}\left(\gamma^{\prime}, \gamma_{+}^{\prime \prime \prime}\right), \quad b=\operatorname{det}\left(\gamma^{\prime}, \gamma_{-}^{\prime \prime \prime}\right), \quad c=-\frac{3 \operatorname{det}\left(\gamma^{\prime}, \gamma^{\prime \prime}\right)\left\langle\gamma^{\prime}, \gamma^{\prime \prime}\right\rangle}{s^{2}} .
$$

Since the one-sided markers are mutually reciprocal, see (3.7), here $\mu=\lambda^{-1}$, then by Lemma 5.1 the right marker $\mu$ is the root of adjoint equation (5.4). Hence, it satisfies identity (5.5).

Corollary 5.3. Under the assumptions of Theorem 4.1 the right marker $\mu=t_{1}^{\prime}\left(t_{0}+0\right)$ of the pseudo-vertex satisfies the identity

$$
\left(\mu^{3}-3 \mu^{2}\right) k_{-}^{\prime}+(3 \mu-1) k_{+}^{\prime}=0 .
$$

Proof. By Corollary 5.2, the right marker $\mu$ satisfies identity (5.5). We note that identities (4.4) and (5.5) imply one the other by the cyclic change of quantities $\lambda, \gamma_{+}^{\prime \prime \prime}, \gamma_{-}^{\prime \prime \prime}$ by $\mu, \gamma_{-}^{\prime \prime \prime}$, $\gamma_{+}^{\prime \prime \prime}$, respectively. Then, making algebraic transformations with (5.5) similar to ones make in Corollary 5.1 with identity (4.4), we obtain (5.6).

Corollary 5.4. Under the assumptions of Theorem 4.1 there exist strictly separated from zero coefficients of the convex combination

$$
\alpha>0, \quad \beta>0, \quad \alpha+\beta=1,
$$

such that

$$
\begin{aligned}
& \alpha \operatorname{det}\left(\gamma^{\prime}, \gamma_{+}^{\prime \prime \prime}\right)+\beta \operatorname{det}\left(\gamma^{\prime}, \gamma_{-}^{\prime \prime \prime}\right)=\frac{3 \operatorname{det}\left(\gamma^{\prime}, \gamma^{\prime \prime}\right)\left\langle\gamma^{\prime}, \gamma^{\prime \prime}\right\rangle}{s^{2}} \\
& \alpha k_{+}^{\prime}+\beta k_{-}^{\prime}=0
\end{aligned}
$$

Proof. The coefficients can be found constructively. We consider identity (4.4). According to Newton binomial,

$$
(\lambda-1)^{3}=\lambda^{3}-3 \lambda^{2}+3 \lambda-1=\lambda^{2}(\lambda-3)+(3 \lambda-1) .
$$

Since the left marker satisfies $\lambda \leqslant 0$, then

$$
(\lambda-1)^{3}<0, \quad \lambda^{2}(\lambda-3)<0, \quad(3 \lambda-1)<0 .
$$

We divide both sides of identity (4.4) by $(\lambda-1)^{3}<0$ and normalize in this way the coefficients at the determinants $\operatorname{det}\left(\gamma^{\prime}, \gamma_{+}^{\prime \prime \prime}\right)$ and $\operatorname{det}\left(\gamma^{\prime}, \gamma_{-}^{\prime \prime \prime}\right)$ :

$$
\frac{\lambda^{2}(\lambda-3)}{(\lambda-1)^{3}} \operatorname{det}\left(\gamma^{\prime}, \gamma_{+}^{\prime \prime \prime}\right)+\frac{3 \lambda-1}{(\lambda-1)^{3}} \operatorname{det}\left(\gamma^{\prime}, \gamma_{-}^{\prime \prime \prime}\right)=\frac{3 \operatorname{det}\left(\gamma^{\prime}, \gamma^{\prime \prime}\right)\left\langle\gamma^{\prime}, \gamma^{\prime \prime}\right\rangle}{s^{2}}
$$


We then obtain expansion (5.7) with convex strictly positive coefficients depending on the left marker. Here

$$
\alpha=\alpha(\lambda)=\frac{\lambda^{2}(\lambda-3)}{(\lambda-1)^{3}}>0, \quad \beta=\beta(\lambda)=\frac{3 \lambda-1}{(\lambda-1)^{3}}>0, \quad \alpha+\beta=1 .
$$

Identities (5.8) are obtained by dividing both sides of identity (5.1) by the same normalizing coefficient $(\lambda-1)^{3}<0$. At that, the coefficients at one-sided curvatures coincide with corresponding coefficients in (5.9).

Considering (5.5), we find its representation as convex combination depending on the right marker $\mu$. We divide both sides of this identity by the normalizing term

$$
(\mu-1)^{3}=\mu^{3}-3 \mu^{2}+3 \mu-1=\mu^{2}(\mu-3)+(3 \mu-1) .
$$

Since the right marker satisfies $\mu \leqslant 0$, then

$$
(\mu-1)^{3}<0, \quad \mu^{2}(\mu-3)<0, \quad 3 \mu-1<0 .
$$

We obtain:

$$
\frac{3 \mu-1}{(\mu-1)^{3}} \operatorname{det}\left(\gamma^{\prime}, \gamma_{+}^{\prime \prime \prime}\right)+\frac{\mu^{2}(\mu-3)}{(\mu-1)^{3}} \operatorname{det}\left(\gamma^{\prime}, \gamma_{-}^{\prime \prime \prime}\right)=\frac{3 \operatorname{det}\left(\gamma^{\prime}, \gamma^{\prime \prime}\right)\left\langle\gamma^{\prime}, \gamma^{\prime \prime}\right\rangle}{s^{2}}
$$

We denote

$$
\widetilde{\alpha}=\widetilde{\alpha}(\mu)=\frac{3 \mu-1}{(\mu-1)^{3}}, \quad \widetilde{\beta}=\widetilde{\beta}(\mu)=\frac{\mu^{2}(\mu-3)}{(\mu-1)^{3}} .
$$

Here $\widetilde{\alpha}>0, \widetilde{\beta}>0, \widetilde{\alpha}+\widetilde{\beta}=1$. Formula 5.10 represents the main identity in Theorem 4.1 by means of convex coefficients depending on the right marker. By straightforward substitution of $\mu=\lambda^{-1}$ into formulae (5.11) we confirm that $\widetilde{\alpha}(\mu)=\alpha(\lambda), \widetilde{\beta}(\mu)=\beta(\lambda)$, that is, formula (5.10) coincides with (5.7). In the same way we find an identity with one-sided derivatives of the curvature with the coefficients depending on $\mu=\lambda^{-1}$, which is equivalent to identity (5.8):

$$
\widetilde{\alpha} k_{+}^{\prime}+\widetilde{\beta} k_{-}^{\prime}=0 .
$$

The coefficients in (5.12) are calculated by formulae (5.11).

\section{APPENDIX}

As an example, we consider Dirichlet problem (2.1) for the case, when the boundary set $M$ is enveloped by a parametrically defined curve

$$
\Gamma=\left\{\gamma(t) \in \mathbb{R}^{2}: \gamma(t)=\left(\gamma_{1}(t), \gamma_{2}(t)\right), t \in T\right\},
$$

where

$$
\begin{aligned}
& \gamma_{1}(t)=r(t) \cos t, \gamma_{2}(t)=r(t) \sin t, t \in T=[0,2 \pi], \\
& r(t)=\left\{\begin{array}{l}
1.5-0.5 \cos ^{2} 2 t-0.1 \cos ^{3} 2 t, \quad t \in\left[0, \frac{\pi}{4}\right) \cup\left[\frac{3 \pi}{4}, \frac{5 \pi}{4}\right) \cup\left[\frac{7 \pi}{4}, 2 \pi\right], \\
1.5-0.5 \cos ^{2} 2 t+0.4 \cos ^{3} 2 t, \quad t \in\left[\frac{\pi}{4}, \frac{3 \pi}{4}\right) \cup\left[\frac{5 \pi}{4}, \frac{7 \pi}{4}\right) .
\end{array}\right.
\end{aligned}
$$

The boundary $\Gamma=\partial M$ of the boundary set is twice continuously differentiable and has four points corresponding to the moments $t=t_{0} \in T^{0}, T^{0}=\left\{\frac{\pi}{4}, \frac{3 \pi}{4}, \frac{5 \pi}{4}, \frac{7 \pi}{4}\right\}$, at which the smoothness of the curvature is broken. These points are the pseudo-vertices of the boundary set. By means of analytic calculations one can easily establish that they are non-stationary in both coordinates, and the one-sided third derivatives at these points are finite and do not coincide; here we have a discontinuity with a finite jump of the third order derivative of the function of the polar radius $r(t)$. Thus, all assumptions of Theorem 4.1 are satisfied. As an 


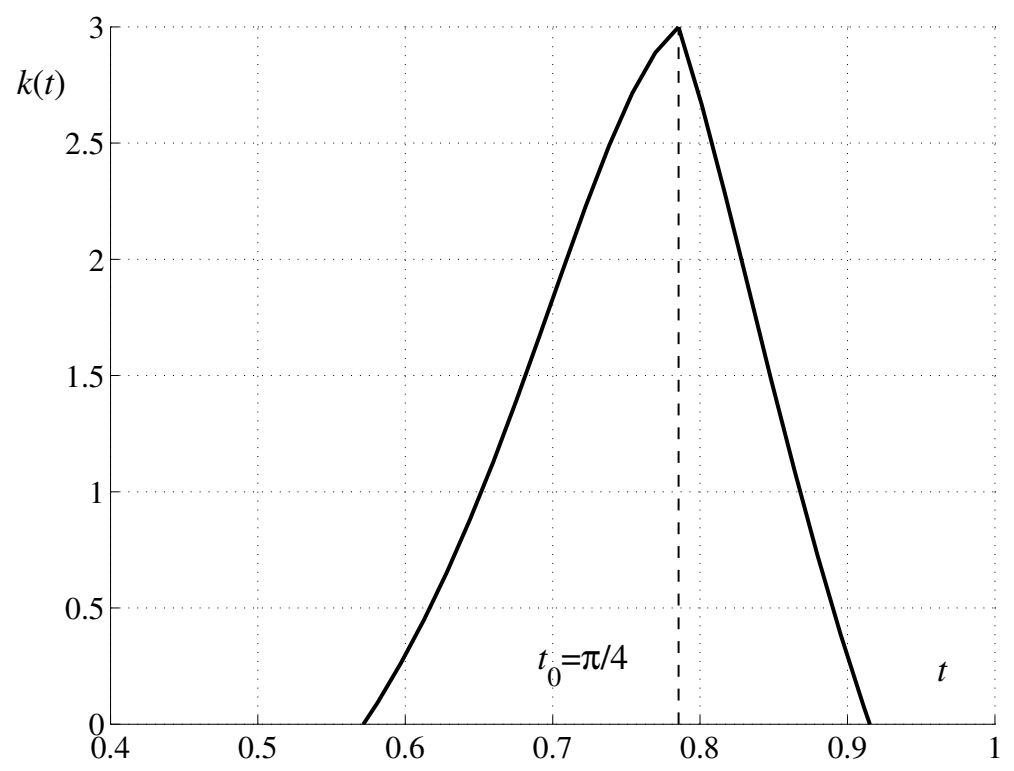

Figure 1: Graph of the curvature $k(t)$ in the vicinity of the pseudo-vertex at $t_{0}=\pi / 4$

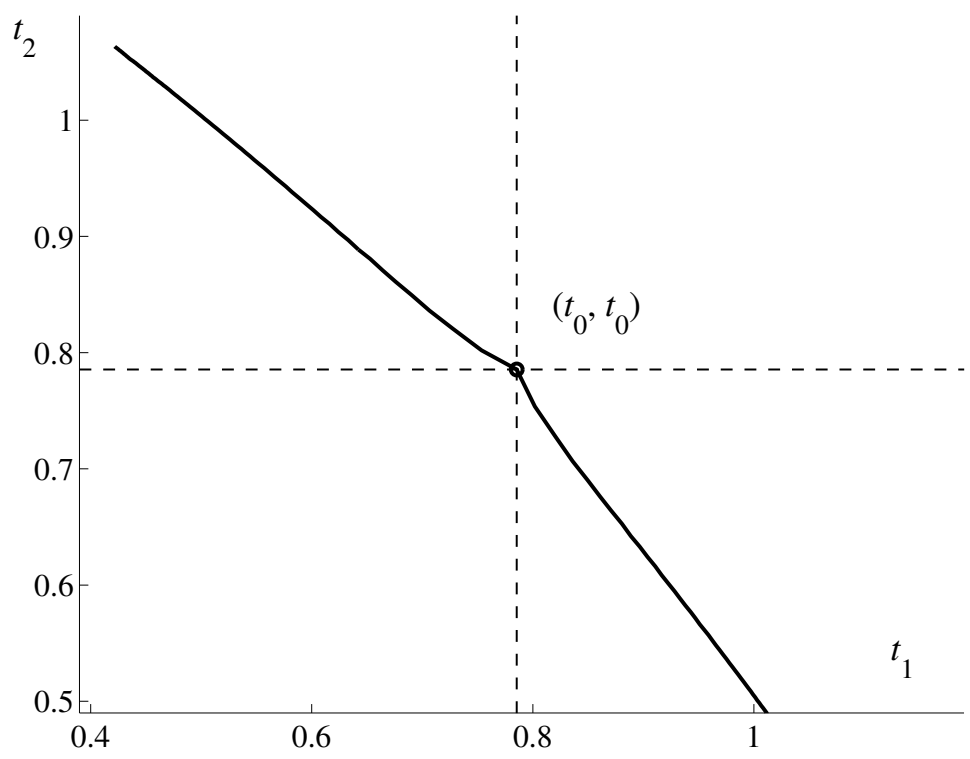

Figure 2: Gluing of graphs of local diffeomorphisms $t_{2}=t_{2}\left(t_{1}\right)$ and $t_{1}=t_{1}\left(t_{2}\right)$ determining the pseudo-vertex of the curve $\Gamma=\partial M$ as $t_{0}=\pi / 4$

illustration, on figure 1 we show that graph of the curvature $k(t)$ in the vicinity of one of the pseudo-vertices as $t_{0}=\pi / 4$. For this point $\gamma_{1}^{\prime}\left(t_{0}\right)=-1 / \sqrt{2} \neq 0, \gamma_{2}^{\prime}\left(t_{0}\right)=1 / \sqrt{2} \neq 0$, the curvature is continuous and its value reads as $k\left(t_{0}\right)=3$. At this point the curvature is a non-smooth function, the one-sided derivatives of the curvature are given by the identities $k^{\prime}\left(t_{0}-0\right)=4.8, k^{\prime}\left(t_{0}+0\right)=-19.2$.

We mention that it is quite complicated to find exactly local diffeomorphisms determining the pseudo-vertices. We find them by numerical-analytic methods; their description is given, for instance, in [15]. The graphs of mutually inverse local diffeomorphisms $t_{2}=t_{2}\left(t_{1}\right)$ and $t_{1}=t_{1}\left(t_{2}\right)$ determining the pseudo-vertex at $t_{0}=\pi / 4$ are provided on Figure 2 . 


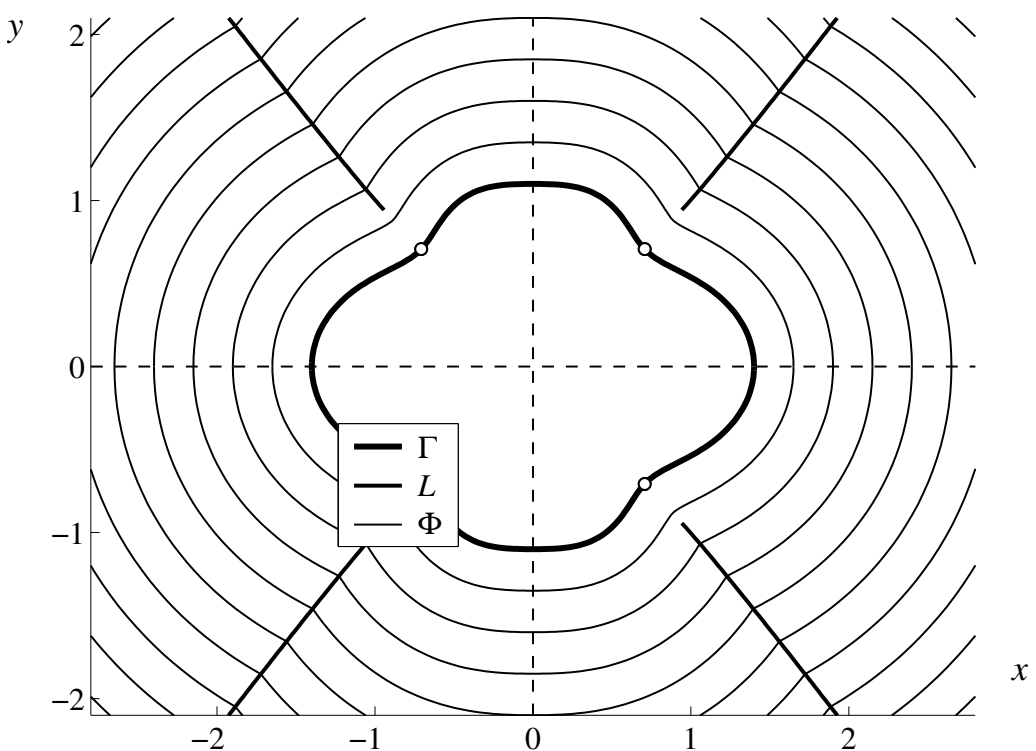

Figure 3: The boundary $\Gamma$ of the boundary set, singular set $L$ and level lines $\Phi$ of the minimax solution $u(\mathbf{x})$; the pseudo-vertices of the set are graphically indicated by circles of a small radius.

It is important to stress that in order to construct the branches of the singular set, at each pseudo-vertex we need to find the value of at least one of two one-sided markers. Here we employ equation (5.1) for finding the left marker. For instance, at the pseudo-vertex corresponding to $t_{0}=\pi / 4$, under known values of one-sided derivatives of the curvature, equation (5.1) becomes

$$
-19.2\left(\lambda^{3}-3 \lambda^{2}\right)+4.8(3 \lambda-1)=0,
$$

and after a simplification,

$$
\lambda^{3}-3 \lambda^{2}-0.75 \lambda+0.25=0 .
$$

An array of roots of the equations found by approximate methods reads as

$$
\Lambda=\{-0.4028,0.19339,3.20942\} .
$$

The value of the left marker of the pseudo-vertex is equal to the negative root, here

$$
\lambda \approx-0.4028 \text {. }
$$

From the geometrical point of view, the left marker is equal to the tangent of slope of the graph $t_{2}=t_{2}\left(t_{1}\right)$ with respect to the positive direction of the axis $t_{1}$. Respectively, the right marker $\mu=\frac{1}{\lambda} \approx-2.4826$ is the tangent of slope of the graph $t_{1}=t_{1}\left(t_{2}\right)$ with respect to the positive direction of the axis $t_{2}$.

Then, knowing the markers and corresponding local diffeomorphisms for each vertex, we construct the branches of the singular set by solving system of equations (3.4). Another approach for constructing the branches of the singular set is related with integrating ordinary differential equations, the boundary conditions for which are determined by the markers of the pseudovertices, while the dynamics is determined by the local diffeomorphisms, see [15]. On Figure 3 we show the branches of the singular set and the level lines map of the minimax solution of problem (2.1) found by approximate methods. On Figure 4 we provide an approximation of the graph of the minimax solution, which, we observe, has a gradient catastrophe at the points of the singular set. 


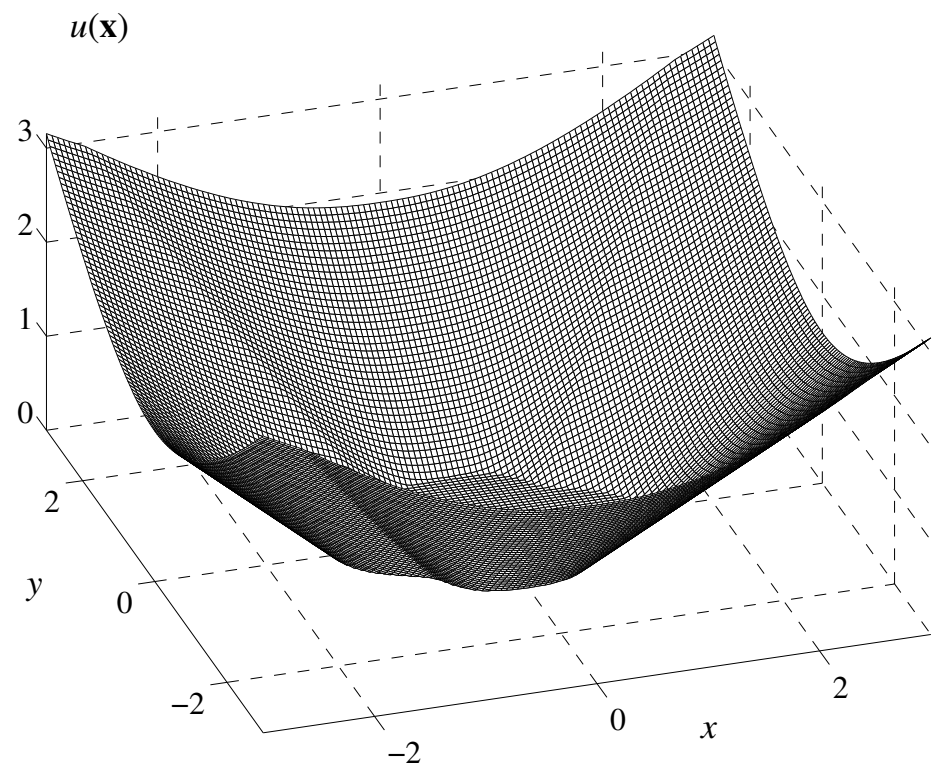

Figure 4: Graph of the minimax solution $u(\mathbf{x})$

\section{BIBLIOGRAPHY}

1. O.A. Oleinik. Discontinuous solutions of non-linear differential equations // Uspekhi Matem. Nauk. 12:3(75), 3-73 (1957). (in Russian).

2. S.N. Kruzhkov. Generalized solutions of the Hamilton-Jacobi equations of eikonal type. I. Formulation of the problems; existence, uniqueness and stability theorems; some properties of the solutions // Matem. Sborn. 98:3, 450-493 (1975). [Math. USSR-Sb. 27:3, 406-446 (1975).]

3. M.G. Crandall, P.L. Lions. Viscosity solutions of Hamilton-Jacobi equations // Trans. Amer. Math. Soc. 277:1, 1-42 (1983).

4. A.I. Subbotin. Generalized solutions of first-order PDEs. The dynamical optimization perspective. Inst. Comp. Tekn., Moscow (2003). [Birkhäuser, Basel (1994).]

5. N.N. Krasovskij, A.I. Subbotin. Game-theoretical control problems Nauka, Moscow (1974). [Springer-Verlag, New York (1988).]

6. G.V. Papakov, A.M. Taras'ev, A.A. Uspenskij. Numerical approximations of generalized solutions of the Hamilton-Jacobi equations // Prikl. Matem. Mekh. [J. Appl. Math. Mech. 60:4, 567-578 (1996).]

7. E.K. Kostousova. On feedback target control for uncertain discrete-time systems through polyhedral techniques // Vychisl. Tekhnol. 22:2, 19-36 (2017). (in Russian).

8. B.I. Ananiev, M.I. Gusev, T.F. Filippova. Control and estimate of states of dynamical systems with uncertanity. Izd-vo SO RAN, Novosibirsk (2018). (in Russian).

9. V.N. Ushakov, A.A. Uspenskii, P.D. Lebedev. Construction of a minimax solution for an eikonaltype equation // Proc. Steklov Inst. Math. 263:2, 191-201 (2008).

10. P.D. Lebedev, A.A. Uspenskii. Conditions of transversality of branches of solution to nonlinear equation in speed problem with a circular indicatrix // Trudy IMM UrO RAN. 14:4, 82-99 (2008). (in Russian).

11. A.A. Uspenskii, P.D. Lebedev. On the set of limit values of local diffeomorphisms in wavefront evolution // Proc. Steklov Inst. Math. 272:1, 255-270 (2011).

12. A.A. Uspenskii. Calculation formulas for nonsmooth singularities of the optimal result function in a time-optimal problem // Trudy Inst. Matem. Mekh. UrO RAN. 20:3, 276-290 (2014). [Proc. Steklov Inst. Math. 291. Suppl. 1. 239-254 (2015).] 
13. A.A. Uspenskii. Necessary conditions for the existence of pseudovertices of the boundary set in the Dirichlet problem for the eikonal equation // Trudy Inst. Matem. Mekh. UrO RAN. 21:1, 250-263 (2015). (in Russian).

14. A.A. Uspenskii, P.D. Lebedev. Identification of singularity of generalized solution to Dirichlet problem for eikonal type equation under minimal smoothness of boundary of boundary set // Vestnik Udmurt. Univ. Matem. Mekh. Komput. Nauki. 28:1, 31-46 (2018). (in Russian).

15. A.A. Uspenskii, P.D. Lebedev. Construction of solution to speed control problem under the smoothness breaking of boundary of target set // Izv. Inst. Matem. Inform. Udmurt. Gosud. Univ. 53, 98-114 (2019). (in Russian).

16. V.N. Ushakov, A.A. Uspenskii. Alpha-set in finite-dimensional Euclidean spaces and their properties // Vestnik Udmurt. Univ. Matem. Mekh. Komput. Nauki. 26:1, 95-120 (2016). (in Russian).

17. J.W. Bruce, P.J. Giblin. Curves and singularities. A geometrical introduction to singularity theory. Cambridge University Press, Cambridge (1984).

18. Th. Bröcker. Differentiable germs and catastrophes. Cambridge Univ. Press, London (1975).

19. A.R. Alimov, I.G. Tsar'kov. Connectedness and solarity in problems of best and near-best approximation // Uspekhi Matem. Nauk. 71:1, 3-84 (2016). [Russ. Math. Surv. 71:1, 1-77 (2016).]

20. N.V. Efimov, S.B. Stechkin. Some properties of Chebyshev sets // Dokl. Akad. Nauk SSSR. 118:1, 17-19 (1958).

21. V.D. Sedykh. On the topology of symmetry sets of smooth submanifolds in $\mathbb{R}^{k} / /$ in "Advanced Studies in Pure Mathematics". 43, 401-419 (2006).

22. D.A. Serkov. On fixed point theory and its applications to equilibrium models // Vestnik YuUrGU. Matem. Model. Program. 9:1, 20-31 (2016). (in Russian).

23. V.I. Arnold. Singularities of caustics and wavefronts. Fazis, Moscow (1996). (in Russian).

24. R. Isaacs. Differential games. A mathematical theory with applications to warfare and pursuit, control and optimization. John Wiley and Sons, New York (1965).

Alexander Alexandrovich Uspenskii,

N.N. Krasovskii Institute of mathematics and mechanics,

S. Kovalevskaya str. 16,

620990, Ekaterinburg, Russia

E-mail: uspen@imm.uran.ru

Pavel Dmitrievich Lebedev,

N.N. Krasovskii Institute of mathematics and mechanics,

S. Kovalevskaya str. 16,

620990, Ekaterinburg, Russia

E-mail: pleb@yandex.ru 